\title{
A Universal Model for Spike-Frequency Adaptation
}

\author{
Jan Benda ${ }^{1} \&$ Andreas V. M. Herz ${ }^{2}$ \\ ${ }^{1}$ Department of Physics, University of Ottawa, Ottawa, Ontario, K1N 6N5, Canada \\ j.benda@biologie.hu-berlin.de \\ ${ }^{2}$ Institute for Theoretical Biology, Humboldt University Berlin, 10115 Berlin, Germany \\ a.herzabiologie.hu-berlin.de
}

Neural Computation 15, 2523-2564 (2003)

Spike-frequency adaptation is a prominent feature of neural dynamics. Among other mechanisms various ionic currents modulating spike generation cause this type of neural adaptation. Prominent examples are voltage-gated potassium currents (M-type currents), the interplay of calcium currents and intracellular calcium dynamics with calciumgated potassium channels (AHP-type currents), and the slow recovery from inactivation of the fast sodium current. While recent modeling studies have focused on the effects of specific adaptation currents, we derive a universal model for the firing-frequency dynamics of an adapting neuron which is independent of the specific adaptation process and spike generator. The model is completely defined by the neuron's onset $f$ - $I$-curve, steady-state $f$ - $I$-curve, and the time constant of adaptation. For a specific neuron these parameters can be easily determined from electrophysiological measurements without any pharmacological manipulations. At the same time, the simplicity of the model allows one to analyze mathematically how adaptation influences signal processing on the single-neuron level. In particular, we elucidate the specific nature of high-pass filter properties caused by spike-frequency adaptation. The model is limited to firing frequencies higher than the reciprocal adaptation time constant and to moderate fluctuations of the adaptation and the input current. As an extension of the model, we introduce a framework for combining an arbitrary spike generator with a generalized adaptation current. 


\section{Introduction}

Spike-frequency adaptation is a widespread neurobiological phenomenon, exhibited by almost any type of neuron that generates action potentials. It occurs in vertebrates as well as in invertebrates, in peripheral as well as in central neurons, and may play an important role in neural information processing. Within the large variety of mechanisms responsible for spike-frequency adaptation ionic currents that influence spike generation are of particular importance. Three main types of such adaptation currents are known: M-type currents, which are caused by voltage-dependent, high-threshold potassium channels (Brown \& Adams, 1980), AHP-type currents, mediated by calciumdependent potassium channels (Madison \& Nicoll, 1984), and slow recovery from inactivation of the fast sodium channel (Fleidervish et al., 1996).

Recent computer simulations and analytical studies have focused on specific adaptation mechanisms (Cartling, 1996; Wang, 1998; Ermentrout, 1998; Ermentrout et al., 2001). To complement these approaches we investigate a large group of potential cellular mechanisms. Our goal is to derive a single universal model that is independent of the biophysical processes underlying adaptation.

Such a framework has various advantages, both from an experimental and a theoretical point of view. For example, it is often desirable to quantify spike-frequency adaptation without performing pharmacological manipulations to characterize specific adaptation currents (Benda et al., 2001). This is particularly true if these currents have not yet been identified in detail. Furthermore, a low-dimensional phenomenological model is well suited for systematic network simulations and may thus help to elucidate the functional role of cellular adaptation on the systems level.

The phenomenon of spike-frequency adaptation is illustrated in Fig. 1. Let us assume that the investigated neuron is in a fully unadapted state. The initial response to a step-like stimulus reflects the properties of the non-adapted cell, which are determined by the fast processes of the spike generator only. The resulting behavior is covered by the neuron's onset $f$ - $I$-curve $f_{0}(I)$ which describes the initial firing frequency $f_{0}$ as a function of the stimulus intensity $I$. Due to adaptation the firing frequency $f$ decays to some steady-state value $f_{\infty}$. The neuron may even stop spiking after a while. Measuring $f_{\infty}$ for different inputs $I$ results in the steady-state $f$-I-curve $f_{\infty}(I)$. Electrophysiological recordings show that the decay of the firing frequency is often approximately exponential and characterized by some effective adaptation time constant $\tau_{\text {eff }}$ which may range from tens of milliseconds (Madison \& Nicoll, 1984; Stocker et al., 1999) to several seconds (Edman et al., 1987; Sah \& Clements, 1999). The model we are going to derive is completely defined by the onset $f$ - $I$-curve $f_{0}(I)$, the steady-state $f$ - $I$-curve $f_{\infty}(I)$, and the effective time constant $\tau_{\text {eff. }}$. These quantities can be easily measured experimentally and thus allow to quickly characterize the adaptation properties of individual neurons.

The paper is organized as follows. In section 2 we extract generic properties of three prototypical adaptation mechanisms. This allows us to derive a universal phenomeno- 

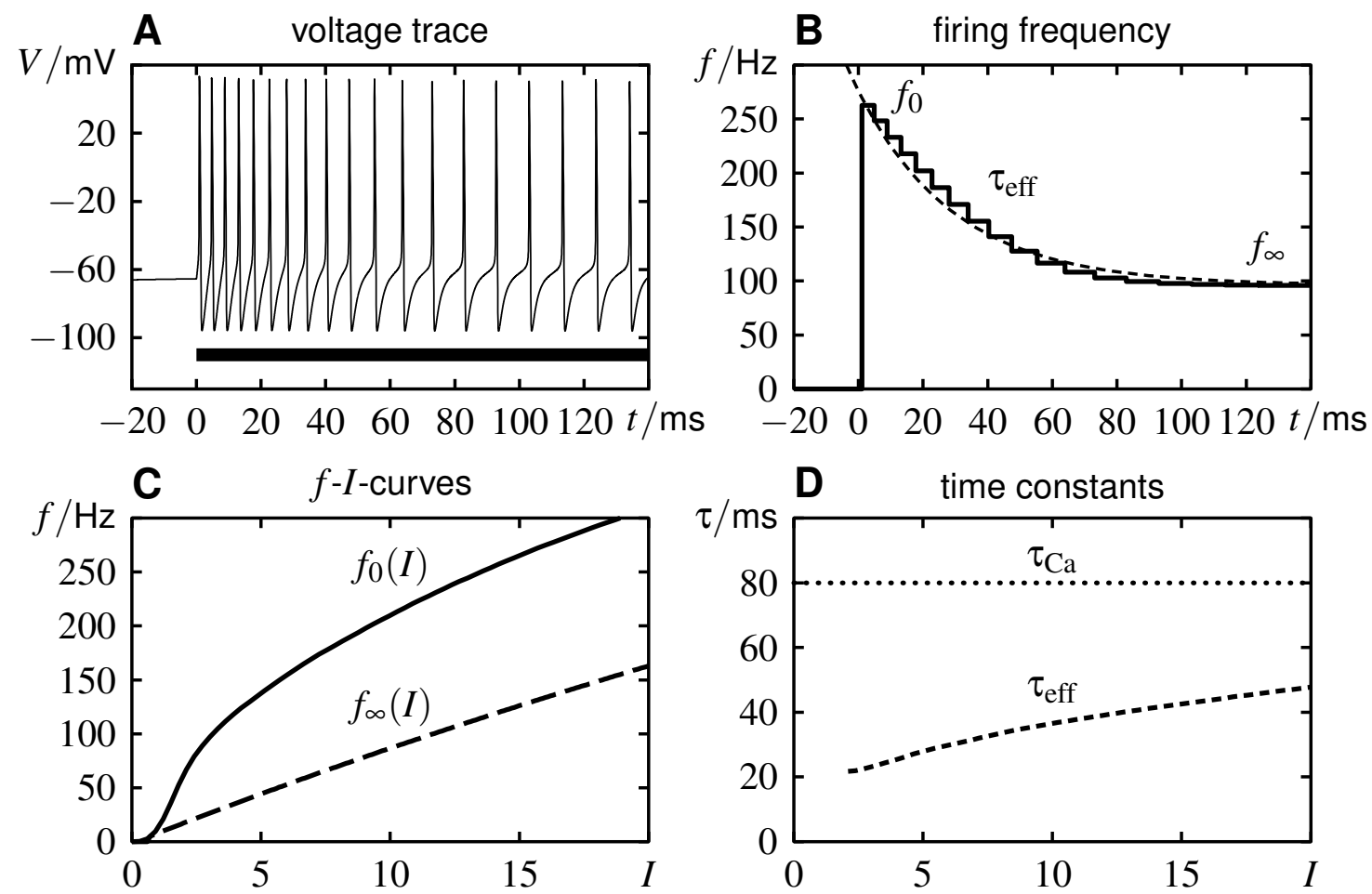

Figure 1: The phenomenon of spike-frequency adaptation. A The voltage trace of a modified Traub-Miles model with mAHP-current (see Appendix for details) evoked by a step-like stimulus $\left(I=18 \mu \mathrm{A} / \mathrm{cm}^{2}\right)$ as indicated by the solid bar. B The corresponding instantaneous firing frequency, defined as the reciprocal of the interspike intervals. The response $f$ decays from its onset value $f_{0}$ in an approximately exponential manner (dashed line) with an effective time constant $\tau_{\text {eff }}$ to a steady-state value $f_{\infty}$. C Measuring the onset and steady-state response at different stimulus intensities results in the onset and the steady-state $f$ - $I$-curves, $f_{0}(I)$ and $f_{\infty}(I)$, respectively. $\mathbf{D} \tau_{\text {eff }}$ depends on input intensity $I$ and is much smaller than the time constant $\tau_{\mathrm{Ca}}$ of the calcium removal which determines the dynamics of adaptation in the model used for this simulation.

logical model in section 3. In section 4 we investigate how the parameters of the model are related to the neuron's $f$ - $I$-curves, and how the adaptation time constant can be estimated experimentally. Based on the model we analyze the effect of adaptation on the neuron's $f$ - $I$-curves and quantify signal transmission properties arising from adaptation in section 5. Section 6 extends our results and shows how the adaptation model can be combined with models of spike generation. We discuss the model in section 7 . A list of commonly used symbols is given in the Appendix.

To illustrate our results we use a modified Traub-Miles model (Ermentrout, 1998) as well as the Crook model (Crook et al., 1998). We add either an M-type current or an mAHP-current to simulate spike-frequency adaptation. The dynamical equations and parameter values are also summarized in the Appendix. 


\section{General characteristics of adaptation currents}

In this section we examine three basic types of ionic currents causing spike-frequency adaptation: M-type currents, mAHP-type currents, and sodium currents with slow recovery from inhibition. Our goal is to show that all these different mechanisms can be described by an effective adaptation current $I_{\mathrm{A}}$ :

$$
\begin{aligned}
I_{\mathrm{A}} & =\bar{g}_{\mathrm{A}} m^{p} h^{q} c a\left(V-E_{\mathrm{A}}\right) \\
\tau_{a}(V) \frac{\mathrm{d} a}{\mathrm{~d} t} & =a_{\infty(V)}-a .
\end{aligned}
$$

As in the following, the time-dependence of dynamical variables has been omitted for simplicity. $\bar{g}_{a}$ is the current's maximum conductance and $E_{\mathrm{A}}$ is its reversal potential. The dynamics (1b) of the adaptation gating variable $a$ is a simple relaxation towards a voltage dependent steady-state variable $a_{\infty(V)}$ with a time constant $\tau_{a}(V)$ that could depend on the membrane potential $V . m$ and $h$ are possible additional voltage gated variables raised to the integer power $p$ and $q$, respectively. Both variables - if present - have to be much faster than the adaptation variable $a$. The constant $c$ is a proportionality factor for $a$. In essence, equations (1) are the well known equations for a voltage gated current as introduced by Hodgkin \& Huxley (1952).

\subsection{M-type currents}

M-type currents are slow voltage dependent potassium currents (Brown \& Adams, 1980). Their dynamics is captured by

$$
\begin{aligned}
I_{\mathrm{M}} & =\bar{g}_{\mathrm{M}} a\left(V-E_{\mathrm{M}}\right) \\
\tau_{a}(V) \frac{\mathrm{d} a}{\mathrm{~d} t} & =a_{\infty(V)}-a,
\end{aligned}
$$

where $\bar{g}_{\mathrm{M}}$ denotes the maximum conductance and $E_{\mathrm{M}}$ the reversal potential. The steady-state variable $a_{\infty(V)}$ is a sigmoidal function of the membrane potential $V$ with values between zero and one. M-type currents are mainly activated during a spike (Fig. 2 and Fig. 3). Between spikes, they deactivate slowly as determined by their time constant $\tau_{a}(V)$. Activation of M-type currents causes spike-frequency adaptation, since as potassium currents they decrease the sensitivity of the spike generator to input currents. Equations (2) are a simple realization of the general description (1) with $a$ being the only gating variable and $c=1$.

\section{2 mAHP-currents}

An important adaptation mechanism arises from medium after-hyperpolarization (mAHP)currents, which are calcium dependent potassium currents (Madison \& Nicoll, 1984). Three processes are involved in this type of adaptation. 

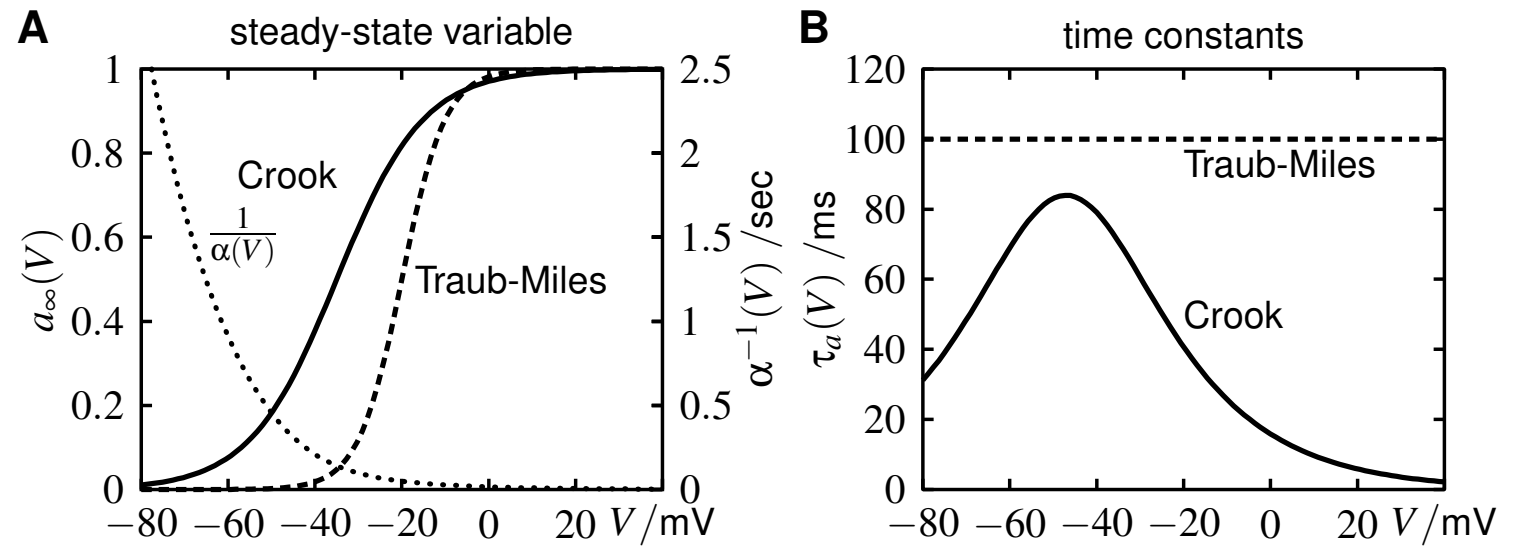

Figure 2: Properties of M-type-currents. A The dependence of the activation function $a_{\infty(V)}$ on the membrane potential $V$ as defined in the Crook model and the modified Traub-Miles model. While in the Crook model the M-type current is slightly activated already at rest $\left(V_{\text {rest }}=-71.4 \mathrm{mV}\right)$, in the modified Traub-Miles model it is only activated during spikes $\left(V_{\text {rest }}=-66.5 \mathrm{mV}\right)$. The dotted line is the inverse rate constant $\alpha(V)$ of the M-type current in the Crook model, given in seconds. B The time constant $\tau_{a}(V)$ in the Crook model is the product of $a_{\infty(V)}$ and $1 / \alpha(V)$ shown in panel A and has a peak within the linear range of $a_{\infty}(V)$. In the modified Traub-Miles model $\tau_{a}(V)$ is assumed to be constant.

First, there are different voltage gated calcium channels (N-, P-, Q-, L- and T-type) that are rapidly activated by depolarizations (about one millisecond, Jaffe et al., 1994). Recent calcium imaging studies show that the total calcium influx per spike is approximately constant (Schiller et al., 1995; Helmchen et al., 1996). Calcium-induced calcium release may also contribute to spike triggered calcium transients (Sandler \& Barbara, 1999). All these processes are very fast. They can be viewed as part of the spike generator and do not lead to adaptation. In the context of adaptation the only relevant effect of these currents is that they increase the intracellular calcium concentration.

Second, calcium is removed with a slow time constant $\tau_{\mathrm{Ca}}$. This process is the result of buffering, diffusion, and calcium pumps and can be described by

$$
\tau_{\mathrm{Ca}} \frac{\mathrm{d}\left[\mathrm{Ca}^{2+}\right]}{\mathrm{d} t}=\beta I_{\mathrm{Ca}}-\left[\mathrm{Ca}^{2+}\right]
$$

i.e. the concentration of intracellular calcium $\left[\mathrm{Ca}^{2+}\right]$ is increased proportionally to the calcium influx $I_{\mathrm{Ca}}$ (Traub et al., 1991). The time constant $\tau_{\mathrm{Ca}}$ of the calcium removal determines the time scale of this type of adaptation. Thus, the calcium dynamics (3) is equivalent to the dynamics (2b) of the gating variable $a$ of an M-type current.

Finally, a potassium current $I_{\mathrm{AHP}}$ is activated depending on the intracellular calcium 
concentration (Brown \& Griffith, 1983; Madison \& Nicoll, 1984):

$$
\begin{aligned}
I_{\mathrm{AHP}} & =\bar{g}_{\mathrm{AHP}} q\left(V-E_{\mathrm{K}}\right) \\
\tau_{q}\left(\left[\mathrm{Ca}^{2+}\right]\right) \frac{\mathrm{d} q}{\mathrm{~d} t} & =q_{\infty}\left(\left[\mathrm{Ca}^{2+}\right]\right)-q
\end{aligned}
$$

This mAHP-current is responsible for spike-frequency adaptation. Due to the slow calcium dynamics (3) $q_{\infty}\left(\left[\mathrm{Ca}^{2+}\right]\right)$ is also changing slowly. The time constant $\tau_{q}$, however, is much smaller than the time constant $\tau_{\mathrm{Ca}}$ of the calcium removal. Thus we can approximate the gating variable $q$ by its steady-state variable $q_{\infty}\left(\left[\mathrm{Ca}^{2+}\right]\right)$. As the analysis of various models shows, $q_{\infty}\left(\left[\mathrm{Ca}^{2+}\right]\right)$ is well captured by a first order Michaelis-Mentenfunction and takes only small values (Crook et al., 1998; Ermentrout, 1998). Therefore we can approximate it by $q_{\infty}\left(\left[\mathrm{Ca}^{2+}\right]\right) \approx c \cdot\left[\mathrm{Ca}^{2+}\right]$ where $c>0$.

With these approximations an mAHP-type current can be summarized as

$$
\begin{aligned}
I_{\mathrm{AHP}} & \approx \bar{g}_{\mathrm{AHP}} c\left[\mathrm{Ca}^{2+}\right]\left(V-E_{\mathrm{K}}\right) \\
\tau_{\mathrm{Ca}} \frac{\mathrm{d}\left[\mathrm{Ca}^{2+}\right]}{\mathrm{d} t} & =\beta I_{\mathrm{Ca}}(V)-\left[\mathrm{Ca}^{2+}\right] .
\end{aligned}
$$

Since the calcium currents are fast, the calcium influx $I_{\mathrm{Ca}}$ has been approximated by a function directly depending on the membrane potential. The dynamics of mAHP-type currents are thus formally equal to those of an M-type current.

\subsection{Slow recovery from inactivation}

Slow recovery from inactivation of fast sodium channels is caused by an additional inactivation of the sodium current, which is much slower than the Hodgkin-Huxleytype inactivation $h$. It induces a use-dependent removal of excitable sodium channels and results in spike-frequency adaptation (Fleidervish et al., 1996).

Such currents are gated by an activation variable $m$ and inactivation variable $h$, and an additional slow inactivation variable $s$ :

$$
\begin{aligned}
I_{\mathrm{Na}} & =\bar{g}_{\mathrm{Na}} m^{3} h s\left(V-E_{\mathrm{Na}}\right) \\
\tau_{m}(V) \frac{\mathrm{d} m}{\mathrm{~d} t} & =m_{\infty}(V)-m \\
\tau_{h}(V) \frac{\mathrm{d} h}{\mathrm{~d} t} & =h_{\infty}(V)-h \\
\tau_{s}(V) \frac{\mathrm{d} s}{\mathrm{~d} t} & =s_{\infty}(V)-s .
\end{aligned}
$$

The time constant $\tau_{m}$ of the activation variable $m$ is shorter than one millisecond and $\tau_{h}$ is of the order of a few milliseconds (Hodgkin \& Huxley, 1952; Martina \& Jonas, 1997). In contrast, the time constant $\tau_{s}$ of the slow inactivation process $s$ ranges from 
a few 100 ms (Martina \& Jonas, 1997; Fleidervish et al., 1996) to more than a second (Edman et al., 1987; French, 1989).

Substituting the term $(1-a)$ for the slow inactivation gating variable $s$ results in

$$
\begin{aligned}
I_{\mathrm{Na}} & =\bar{g}_{\mathrm{Na}} m^{3} h\left(V-E_{\mathrm{Na}}\right)-\bar{g}_{\mathrm{Na}} m^{3} h a\left(V-E_{\mathrm{Na}}\right) \\
\tau_{s}(V) \frac{\mathrm{d} a}{\mathrm{~d} t} & =1-s_{\infty}(V)-a
\end{aligned}
$$

By this transformation, we have formally split $I_{\mathrm{Na}}$ into two components. The first one depends only on the two fast gating variables $m$ and $h$, and is responsible for spike initiation only. The second component depends on the two fast gating variables $m$ and $h$ and on the gating variable $a$. The time constant $\tau_{s}(V)$ of the dynamics (7b) of $a$ is voltage dependent and much slower than the spike generator. The steady-state variable $1-s_{\infty}(V)$ is mainly activated at depolarized potentials, i.e. during spikes. Thus, this second component causes adaptation. It conforms with the general adaptation current (1a) with $c=-1$. The dynamics (7b) resembles that of an M-type current (2b).

The adaptation current differs from the spike-initiating component in (7a) by the factor $a$. Under realistic conditions $a$ never gets close to its maximum value, which is unity, since very high sustained firing frequencies would be required to do so. Therefore, most of the time the adaptation current is smaller than the spike-initiating component. Because $V$ stays always below the reversal potential of the sodium current, the driving force $V-E_{\mathrm{Na}}$ is negative so that the second component in (7a) is positive as the M-type current.

\section{Universal phenomenological model}

The previous section has shown that three fundamental adaptation mechanisms can be reduced to a single current (1a) which is gated by a single variable obeying a first order differential equation (1b). We now go one step further and derive a phenomenological model for the firing frequency of an adapting neuron, whose parameter are independent of the specific adaptation process. To achieve this goal we replace the adaptation gating variable $a$ as well as the adaptation current $I_{\mathrm{A}}$ by suitable time averages. All the dependencies on the membrane potential can then be replaced by functions depending on the firing frequency $f$. The resulting universal model for spike-frequency adaptation reads

$$
\begin{aligned}
f & =f_{0}(I-A \cdot[1+\gamma(f)]) \\
\tau \cdot[1+\varepsilon(f)] \frac{\mathrm{d} A}{\mathrm{~d} t} & =A_{\infty}(f)-A .
\end{aligned}
$$

The adaptation state $A$ generalizes the averaged adaptation gating variable $a$ and decays with the adaptation time constant $\tau$ towards the steady-state adaptation strength $A_{\infty}$ 
which depends on the current firing frequency $f$. The averaged adaptation current $A \cdot[1+\gamma(f)]$ depends linearly on $A$ and may be influenced by $f$ through $\gamma(f)$. The term $\varepsilon(f)$ covers a potential dependence of $\tau$ on $f$. The input current $I$ minus the averaged adaptation current is mapped trough the neuron's onset $f-I$-curve $f_{0}(I)$ to result in the firing frequency $f$.

In the next subsection we first motivate equation (8a). We then derive the simplified adaptation current and its dynamics (8b) from the general adaptation current (1).

\subsection{Spike generator and firing frequency}

Let us first consider a spiking neuron which does not adapt at all. The neuron only contains fast ion channels responsible for spike generation. The membrane potential $V$ at the neuron's spike initiating zone evolves according to

$$
C \frac{\mathrm{d} V}{\mathrm{~d} t}=-\sum_{i} g_{i}\left(V-E_{i}\right)+I .
$$

The parameter $C$ is the membrane capacitance. Ionic currents of type $i$ are characterized by a reversal potential $E_{i}$ and a conductance $g_{i}$, whose dynamics is described by further differential equations (Hodgkin \& Huxley, 1952; Johnston \& Wu, 1997). The input current $I$ can be viewed as a dendritic current, a synaptic current, or as a current injected through a microelectrode.

In general the membrane equation (9) cannot be solved analytically. However, we do not need to know the exact time course of the membrane potential, because we are only interested in times at which spikes occur. For strong enough input the neuron fires repetitively with firing frequency $f$ (Hodgkin, 1948). For constant or slowly varying stimulus $I(t)$, this is captured by the neuron's $f-I$-curve

$$
f(t)=f_{0}(I(t)),
$$

the most simple transformation of an input current into spikes. In the following we use (10) to indicate that the spike generator transforms the input signal into a sequence of spikes from which a firing frequency $f(t)$ can be computed. We discuss this process and the validity of (10) in more detail in section 6 . The main advantage of using the neuron's $f$-I-curve to characterize its encoding properties is that for real neurons the $f$ - $I$-curve can be easily obtained from electrophysiological recordings.

Adding an adaptation current (1a) can be viewed as adding a second input current. Formally, the firing frequency of the neuron is then given by

$$
f=f_{0}\left(I-I_{\mathrm{A}}\right)=f_{0}\left(I-\bar{g}_{\mathrm{A}} m^{p} h^{q} c a\left(V-E_{\mathrm{A}}\right)\right) .
$$

This provides a first hint that the main effect of an adaptation current may be a shift of the neuron's $f$-I-curve in the direction of higher input currents $I$. 
Equation (11) is, however, insufficient for a model that involves firing frequency only, since it still contains $m, h$, and $V$. As a next step we show how the adaptation current can be replaced by a suitable average which no longer depends on the spike generator.

\subsection{Averaging the adaptation current}

Since the overall evolution of the adaptation gating-variable $a$ is slow compared to spike generation (see for example Fig. 3C), we may try to separate both sub-systems and replace $a$ by its running average $\langle a\rangle_{T}$ over one period $T$ of the fast sub-system

$$
a(t) \approx\langle a\rangle_{T}(t):=\frac{1}{T(t)} \int_{t-T(t) / 2}^{t+T(t) / 2} a\left(t^{\prime}\right) \mathrm{d} t^{\prime}
$$

where $T(t)$ denotes the time-dependent interspike interval (ISI). To allow this distinction between a fast and a slow dynamics, $T(t)$ has to be short compared to the time constant of the adaptation processes, which is true for sufficiently high firing frequencies. This key assumption implies that the spike generator is operating in its super-threshold regime.

We next aim at replacing the adaptation current $I_{A}$ in (1a) by a suitable average

$$
\left\langle I_{A}\right\rangle_{T, w}=\int_{0}^{T} w(t) I_{A}(t) \mathrm{d} t,
$$

where the normalized weight function $w(t), \int_{0}^{T} w \mathrm{~d} t=1$, is chosen such that $\left\langle I_{A}\right\rangle_{T, w}$ does not change the effect on the resulting firing frequency. Inserting the general adaptation current (1a) and replacing $a$ by its time average (12) we obtain

$$
\left\langle I_{A}\right\rangle_{T, w}=\left\langle\bar{g}_{\mathrm{A}} m^{p} h^{q} c\langle a\rangle_{T}\left(V-E_{\mathrm{A}}\right)\right\rangle_{T, w}
$$

where we can move $\bar{g}_{\mathrm{A}} c\langle a\rangle_{T}$ out of the average. Then (14) represents an average over the variables $V, m$, and $h$ of the spike generator only.

If the effect of the adaptation current on the time course of these variables is approximately independent of the specific value of $\langle a\rangle_{T}$, then there exists a weight function which is independent of adaptation, i.e. the weight is solely a property of the spike generator. This is the second assumption needed for the separation of the fast spiking and the slow adaptation dynamics. It implies that the adaptation current simply reduces the input current and that fluctuations of the adaptation current have a negligible effect on the time course of the spike generator. This assumption amounts to a weak coupling between the adaptation current and the spike generator. Its validity depends on the particular dynamics of the spike generator and on the strength of the adaptation current as 


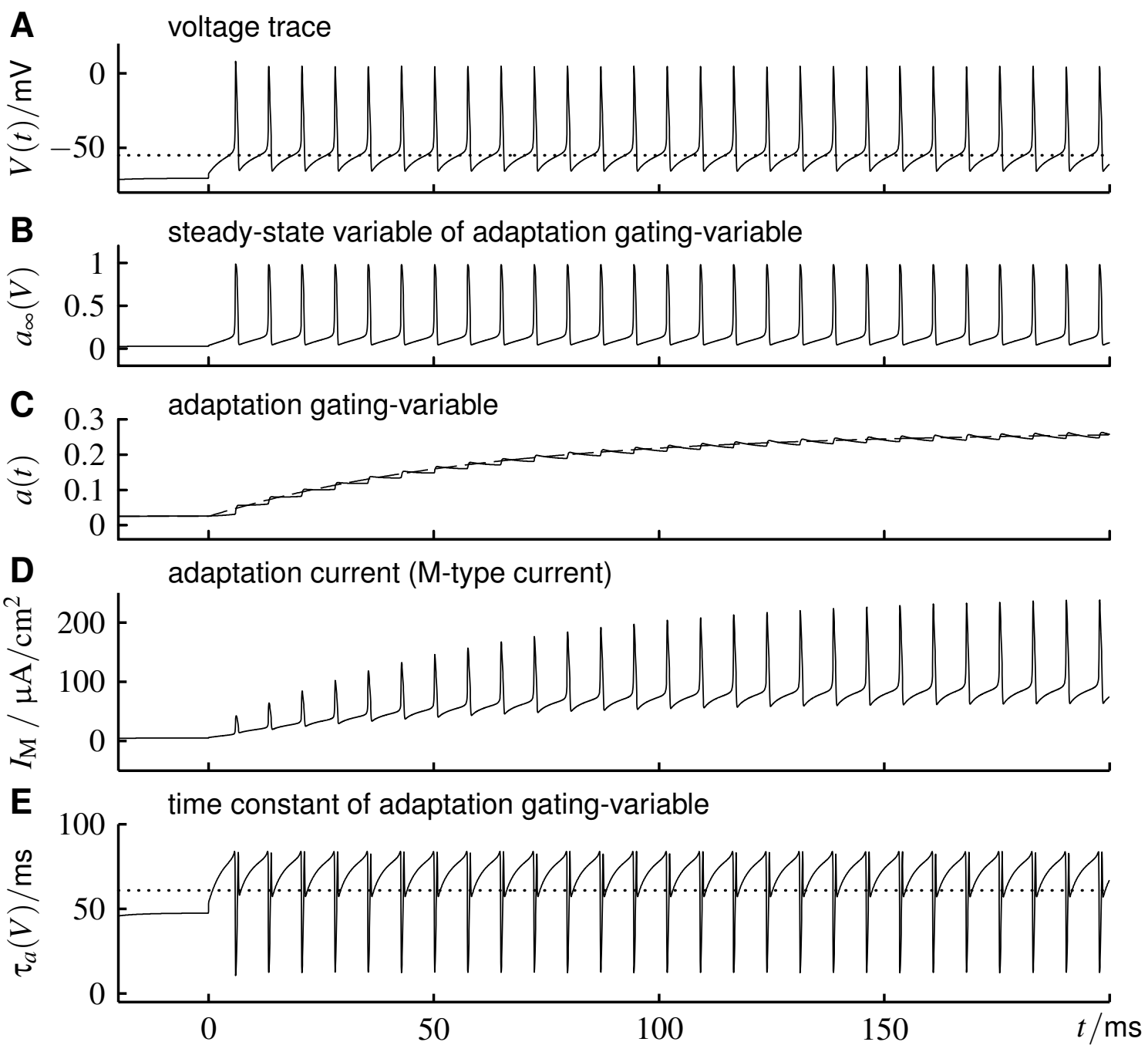

Figure 3: The dynamics of an adaptation current. Shown is a simulation of the Crook model stimulated with a constant current $I=4 \mu \mathrm{A} / \mathrm{cm}^{2}$ starting at $t=0$. Only the sodium, potassium and calcium currents are included in the membrane equation so that the firing frequency is constant and does not adapt. To illustrate the generic behavior of adaptation currents, panels $B$ - E display the dynamical variables of an M-type current activated by the voltage trace shown in A. The steady-state variable $a_{\infty(V)}$ and the time constant $\tau_{a}(V)$ used to model the M-type current are shown in Fig. 2. A The voltage trace. The dotted straight line marks the potential above which $a_{\infty(V)}$ is activated. B The time course of $a_{\infty(V)}$ resulting from the voltage trace in A. C Due to the fast deflections of $a_{\infty(V)}$ the adaptation gating-variable $a$ increases rapidly during spikes. Between the spikes $a$ decays with the time constant $\tau_{a}(V)$ shown in E. The time course of $a$ can be well approximated by its running average $\langle a\rangle_{T}$, which is roughly exponential (dashed line) with a time constant of $61 \mathrm{~ms}$ in this simulation. D The adaptation current $I_{\mathrm{M}}=\bar{g}_{\mathrm{M}} a\left(V-E_{\mathrm{M}}\right)$. Note its large fluctuations caused by the spike activity. $\mathbf{E}$ The time constant $\tau_{a}(V)$ also fluctuates strongly during the spikes. The dotted line denotes the value of the time constant corresponding to the mean gating variable in $\mathrm{C}$. 

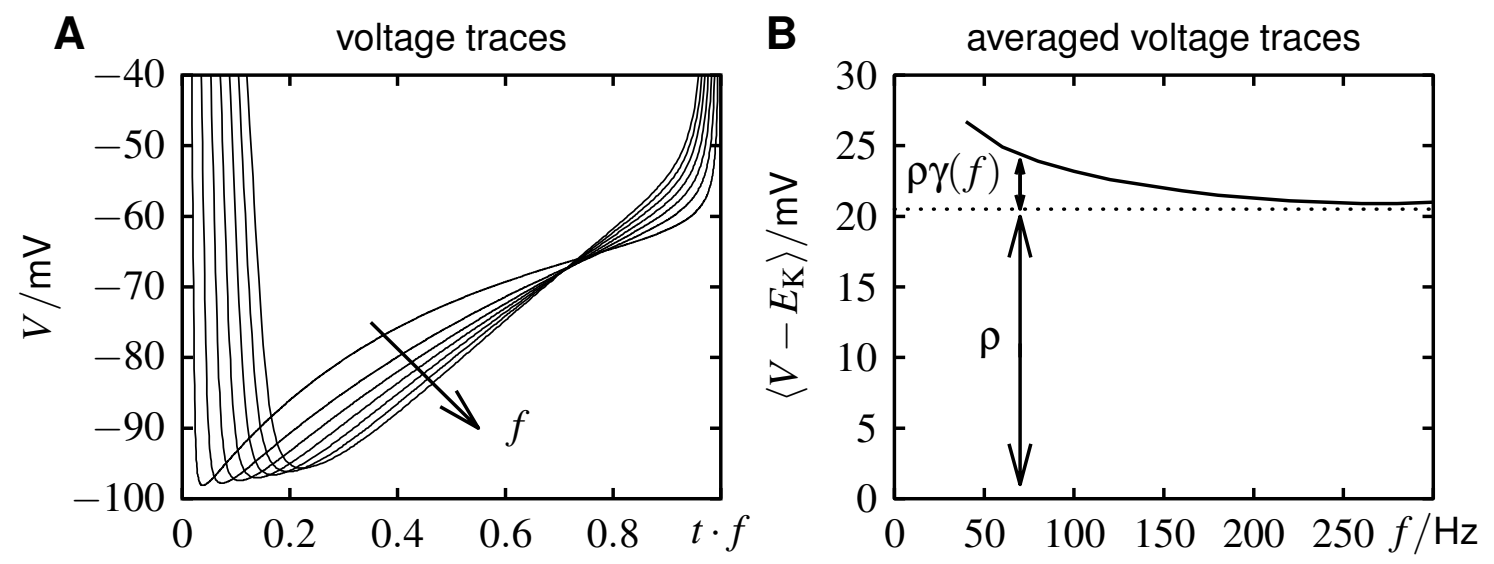

Figure 4: Averaging the membrane potential. A Time course of the membrane potential $V(t)$ during interspike intervals for different firing frequencies $f=40,80,120,160,200,240$, and $280 \mathrm{~Hz}$. Shown are results from the modified Traub-Miles model with mAHP-type current. For low firing frequencies the membrane potential stays longer near about $-70 \mathrm{mV}$. With increasing firing frequency more time is spent at more hyperpolarized potentials. B According to equation (15), the averaged driving force $\left\langle V-E_{\mathrm{K}}\right\rangle_{T, w}$ is a function of the firing frequency (for simplicity we used the response function of the $\theta$-neuron $z(t)=1-\cos (2 \pi t / T)$ (Ermentrout, 1996) for the weight $w$ to generate the data shown in the plot). Its absolute value $\rho$ is larger than the $f$-dependent term $\rho \gamma(f)$.

stronger adaptation currents will also have stronger fluctuations. The potential dependence of the $\gamma(f)$-term in (8a) on $A$ can be used to verify this assumption (see section 4).

In the Appendix we show that for small adaptation strength the weight $w$ in (13) is directly related to the neuron's normalized response function. Response functions are typically small during spikes and deviate strongly from zero between spikes (Reyes \& Fetz, 1993; Hansel et al., 1995; Ermentrout, 1996). Strong fluctuations of the adaptation current during a spike and during the refractory period, as in Fig. 3D, have almost no effect on the firing behavior. What really matters for spike generation is the time course of the adaptation current once the neuron has recovered from the last action potential.

Since in (14) we average over the variables $V, m$, and $h$ of the fast spike-generating dynamics the detailed time course of these fast variables is no longer important. Due to the weak coupling assumption their time course is independent of adaptation and thus is uniquely characterized by the resulting firing frequency, since usually the superthreshold part of $f$ - $I$-curves is strictly monotonic. We can therefore replace the remaining term $\left\langle m^{p} h^{q}\left(V-E_{\mathrm{A}}\right)\right\rangle_{T, w}$ from averaging (1a) by some function $\tilde{\rho}(f)$ :

$$
\left\langle m^{p} h^{q}\left(V-E_{\mathrm{A}}\right)\right\rangle_{T, w} \approx \tilde{\rho}(f) .
$$

The example shown in Fig. 4A illustrates how the time course of the voltage trace 
may depend on $f$. Similar graphs from experimental data can be found in the literature, see e.g. Schwindt (1973). To emphasize the functional form of $\tilde{\rho}(f)$ we rewrite this term as $\rho \cdot[1+\gamma(f)]$, where $\rho$ is a constant and $\gamma(f)$ captures the frequency dependence (Fig. 4B).

The adaptation current can thus be approximated by a function only depending on $f$ :

$$
I_{\mathrm{A}} \approx \bar{g}_{\mathrm{A}} c\langle a\rangle_{T} \rho \cdot[1+\gamma(f)] .
$$

For adaptation based on potassium currents (M-type and mAHP-type currents) both $\rho$, which equals $\left\langle V-E_{\mathrm{K}}\right\rangle_{T, w}$, and $c$ are positive. For slow recovery from inactivation of sodium currents where $c<0$ the membrane potential stays always below the reversal potential $E_{\mathrm{Na}}$ resulting in a negative $\rho$. Thus $c \rho$ is again positive. Defining

$$
A:=\bar{g}_{\mathrm{M}} c\langle a\rangle_{T} \rho
$$

as the adaptation state $A$ and inserting (16) into (11) we finally obtain (8a). Adaptation shifts the onset $f$-I-curve $f_{0}(I)$, as expected from equation (11). The $\gamma(f)$-term adds a complication in that it distorts the $f$-I-curve.

\subsection{Averaging the adaptation dynamics}

It remains to show how the dynamics (8b) for the adaptation state $A$ can be derived from the dynamics (1b) of the adaptation variable $a$. To do so we average (1b) over one ISI to get an equation for $\langle a\rangle_{T}$, which by definition (17) is proportional to $A$.

The possible $V$-dependence of $\tau_{a}(V)$ introduces a complication. If we average equation (1b) directly we have to factorize $\left\langle\tau_{a}(V) \mathrm{d} a / \mathrm{d} t\right\rangle_{T}$ into the product of $\left\langle\tau_{a}(V)\right\rangle_{T}$ and $\langle\mathrm{d} a / \mathrm{d} t\rangle_{T}$ to isolate $\langle a\rangle_{T}$. However, this is poses a problem as $\tau_{a}(V)$ and $\mathrm{d} a / \mathrm{d} t$ covary: According to the Hodgkin-Huxley formalism, $\tau_{a}(V)$ is given by $a_{\infty(V)}$ divided by the corresponding rate constant $\alpha(V)$ of the transition of the channels from their closed to their open state (Johnston \& Wu, 1997). Typically, $\alpha(V)$ increases monotonically and $a_{\infty(V)}$ is a sigmoidal function whose linear range is located above the cell's resting potential and $\tau_{a}(V)$ takes its maximum above but close to the resting potential (see also Fig. 2). This results in a brief but strong negative deflection of $\tau_{a}(V)$ from its mean value during an action potential, as visible in Fig. 3E. At the same time, $a(t)$ increases in a step like manner when a spike occurs. This implies that $\tau_{a}(V)$ and $\mathrm{d} a / \mathrm{d} t$ are strongly anti-correlated. For example, for the Crook model displayed in Fig. 3, the correlation is $r=-0.79$. Thus we cannot average equation (1b) directly and have to search for an alternative approach.

To isolate $\langle a\rangle_{T}$, we divide both sides of (1b) by $\tau_{a}(V)$ and then average over one ISI:

$$
\left\langle\frac{\mathrm{d} a}{\mathrm{~d} t}\right\rangle_{T}=\left\langle\frac{a_{\infty(V)}}{\tau_{a}(V)}\right\rangle_{T}-\left\langle\frac{a}{\tau_{a}(V)}\right\rangle_{T} .
$$


In general, the length of the averaging window $T(t)$ depends on time $t$, so that

$$
\left\langle\frac{\mathrm{d} a}{\mathrm{~d} t}\right\rangle_{T}=\frac{\mathrm{d}\langle a\rangle_{T}}{\mathrm{~d} t}-\frac{\mathrm{d} T / \mathrm{d} t}{T}\left(\frac{a(t+T / 2)+a(t-T / 2)}{2}-\langle a\rangle_{T}\right) .
$$

Since we assume that $a$ changes little during one ISI, we can neglect the last term in parentheses and obtain

$$
\left\langle\frac{\mathrm{d} a}{\mathrm{~d} t}\right\rangle_{T} \approx \frac{\mathrm{d}\langle a\rangle_{T}}{\mathrm{~d} t}
$$

We still have to replace the term $\left\langle a / \tau_{a}(V)\right\rangle_{T}$ by an appropriate factorization. This is possible because the fast fluctuations of $\tau_{a}(t)$ during a spike strongly reduce a possible correlation between $a(t)$ and $1 / \tau_{a}(t)$. In the simulation shown in Fig. 3 this correlation is less than 0.15 . The term $\left\langle a / \tau_{a}(V)\right\rangle_{T}$ may therefore be approximated by $\langle a\rangle_{T}\left\langle 1 / \tau_{a}(V)\right\rangle_{T}$.

Dividing (18) by $\left\langle 1 / \tau_{a}(V)\right\rangle_{T}$ then results in the desired dynamics for $\langle a\rangle_{T}$ :

$$
\frac{1}{\left\langle 1 / \tau_{a}(V)\right\rangle_{T}} \frac{\mathrm{d}\langle a\rangle_{T}}{\mathrm{~d} t}=\frac{1}{\left\langle 1 / \tau_{a}(V)\right\rangle_{T}}\left\langle\frac{a_{\infty(V)}}{\tau_{a}(V)}\right\rangle_{T}-\langle a\rangle_{T} .
$$

As shown for equation (15), we can approximate averages of functions depending on $V$ by functions depending on the firing frequency $f$. Doing so, we obtain the time constant

$$
\tilde{\tau}(f) \approx \frac{1}{\left\langle 1 / \tau_{a}(V)\right\rangle_{T}}
$$

and steady-state variable

$$
\kappa(f) \approx \frac{1}{\left\langle 1 / \tau_{a}(V)\right\rangle_{T}}\left\langle\frac{a_{\infty}(V)}{\tau_{a}(V)}\right\rangle_{T} .
$$

With these abbreviations (21) reads

$$
\tilde{\tau}(f) \frac{\mathrm{d}\langle a\rangle_{T}}{\mathrm{~d} t}=\kappa(f)-\langle a\rangle_{T}
$$

Both $\kappa(f)$ and $\tilde{\tau}(f)$ can be obtained from either the time course of the adaptation gating variable $a$ (Fig. 3C), or by the averages (22) and (23) over a single ISI. Both methods agree well as illustrated in Fig. 5 for the modified Traub-Miles model and the Crook model with M-type currents. It is worthwhile to compare $\tilde{\tau}(f)$ with the time constant governing $\left\langle I_{\mathrm{A}}\right\rangle_{T, w}$. Fig. 5A shows that these two functions agree well, too. Thus, at least for these two models and slowly-varying input currents the approximations involved in the averaging procedure are valid.

As suggested by Fig. 5A variations of $\tilde{\tau}(f)$ might be small compared to its absolute value. Therefore we rewrite $\tilde{\tau}(f)$ as $\tau[1+\varepsilon(f)]$ where $\tau$ is a constant and $\varepsilon(f)$ captures the dependence on the firing frequency. Multiplying (24) with $\bar{g}_{\mathrm{A}} c \rho$ and setting $A_{\infty}(f)=\bar{g}_{\mathrm{A}} c \rho \kappa(f)$, we finally obtain the differential equation (8b) for $A=\bar{g}_{\mathrm{A}} c \rho\langle a\rangle$ (17). 

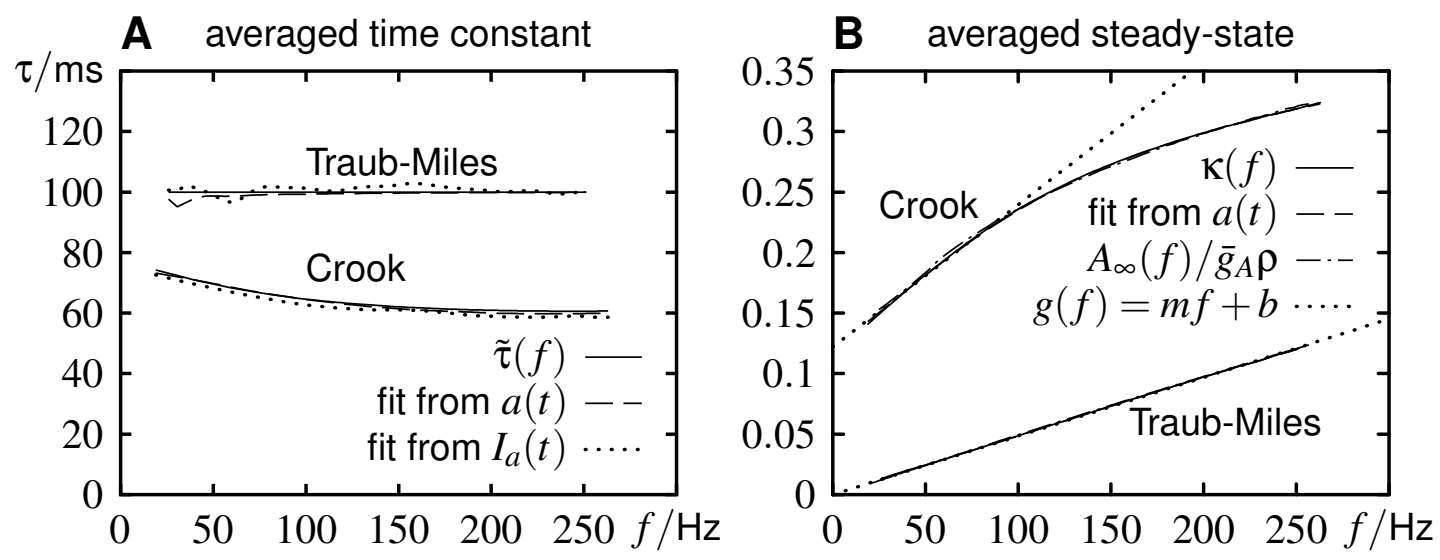

Figure 5: Averaging the dynamics of M-type current gating-variables. Carrying out the same simulation as in Fig. 3 (M-type current not included in the membrane equation) allows us to measure the adaptation time constant $\tilde{\tau}(f)$ and the steady-state variable $\kappa(f)$ as a function of the firing frequency $f$ from the time course of the gating variable $a$. Alternatively, these two quantities can be determined as the averages given in (22) and (23). The graphs show simulations of the modified Traub-Miles model and the Crook model. A The adaptation time constant as the average $\tilde{\tau}(f)=1 /\left\langle 1 / \tau_{a}(V)\right\rangle_{T}$ over a single interspike interval (solid lines), fitted from the time course of the adaptation gating-variable $a(t)$ (dashed lines, see also Fig. 3C), and fitted from the time course of the resulting adaptation current $I_{M}$ (dotted lines, Fig. 3D). All three measures agree well, thus confirming the averaging procedures. B The steady-state adaptation variable as the average $\kappa(f)=1 /\left\langle 1 / \tau_{a}\right\rangle_{T}\left\langle a_{\infty} / \tau_{a}\right\rangle_{T}$ over a single interspike interval (solid lines), measured from the time course of the adaptation gating-variable $a(t)$ (dashed lines), and as $A_{\infty}(f) / \bar{g}_{A} \rho$ determined from the onset and steady-state $f$-I-curve of the models with the Mtype current included using (26) (dashed-dotted lines). The factor $\bar{g}_{A} \rho$ and the necessary offset were chosen to fit $\kappa(f)$. This resulted for the modified Traub-Miles model in $\rho=29 \mathrm{mV}$ and for the Crook model in $\rho=1.7 \mathrm{mV}$. Again, all three measures agree well. For comparison, the best fitting straight lines $g(f)=m f+b$ for low firing frequencies are also plotted (dotted lines). 


\section{Parameters of the adaptation model}

With the exception of the onset $f$-I-curve $f_{0}(I)$ all parameters of the model rely on microscopic properties of a specific adaptation mechanism through averages over the adaptation gating variable or the membrane potential. We next show how the model parameters can be obtained from macroscopic measurements.

\subsection{Steady-state strength of adaptation}

In steady state the firing frequency is given by $f_{\infty}(I)$ and the adaptation state $A$ equals $A_{\infty}$. Solving the equation for the adapted firing frequency (8a) for $A_{\infty}$ results in

$$
A_{\infty}\left(f_{\infty}\right)=\frac{I-f_{0}^{-1}\left(f_{\infty}\right)}{1+\gamma\left(f_{\infty}\right)}
$$

where $f_{0}^{-1}$ is the inverse function of the onset $f$-I-curve $f_{0}$. In steady state the input $I$ obeys $I=f_{\infty}^{-1}\left(f_{\infty}\right)$, so that

$$
A_{\infty}(f)=\frac{f_{\infty}^{-1}(f)-f_{0}^{-1}(f)}{1+\gamma(f)}
$$

In Fig. 5B $A_{\infty}(f)$ is compared with the averaged steady-state gating variable $\kappa(f)$.

What functional behavior do we expect for $A_{\infty}(f)$ ? Recall that $A_{\infty}(f)$ is proportional to $\kappa(f)$. To understand the dependence of $\kappa(f)$ on $f$ we decompose the time course of $a_{\infty}(V(t))$ during one ISI into a stereotypical waveform $a_{S}(t)$ reflecting the spike (with duration $T_{S}$ ) and $a_{I S I}(t)$ describing the non-spike related part of $a_{\infty(V)}$. Assuming $\tau_{a}(V)$ to be constant the average (23) reads

$$
\kappa(f) \approx\left\langle a_{\infty(V)}\right\rangle_{T}=\frac{1}{T}\left(\int_{0}^{T_{S}} a_{S}(t) \mathrm{d} t+\int_{0}^{T} a_{I S I}(t) \mathrm{d} t\right) .
$$

The first integral is a constant since the spike waveform is usually independent of firing frequency. The second integral is small compared to the first one since $a_{\infty(V)}$ is not significantly activated by the low membrane potentials between spikes. We therefore expect $\kappa(f)$ and thus $A_{\infty}(f)$ to be proportional to $f=1 / T$. Deviations from this behavior are caused by an activation of adaptation channels between spikes, or by frequency dependent spike deformations.

For the modified Traub-Miles model $A_{\infty}(f)$ is indeed proportional to $f$, (Fig. 5B), because the M-type current of this model is activated during spikes only (Fig. 2A). In the Crook model, however, the current is already activated at lower potentials. This causes a nonlinear $\kappa(f)$ and a positive offset at $f=0$. The offset can be removed by adding the spike independent part of the M-type current to the membrane equation (9). Doing so, $\kappa(f)$ becomes approximately proportional to $f$ for small firing frequencies. 


\subsection{The $\gamma(f)$ - term}

The $\gamma(f)$-term describes the frequency dependence of the averaged adaptation current (16). To determine this term at least one adapted $f$-I-curve $f(I ; A)$ of the neuron being at a certain constant adaptation state $A$ is needed. $\gamma(f)$ can then be derived from equation (8a),

$$
\gamma(f)=\frac{I-f_{0}^{-1}(f(I ; A))}{A}-1 .
$$

In this equation $A$ is the distance between the onset $f$-I-curve $f_{0}(I)$ and the adapted $f$-I-curve $f(I ; A)$ at some firing frequency. Note that $\gamma(f)$ is small in a region around this firing frequency. It can therefore be neglected for small fluctuations of the input $I$. In Fig. 6 an example of $\gamma(f)$ is shown, together with information about how to measure $f(I ; A)$.

Can we neglect the $\gamma(f)$-term if the input has larger fluctuations? Let us decompose the time course of the membrane potential into a stereotypical spike waveform $V_{S}(t)$ of duration $T_{S}$ and a second term $V_{I S I}(t)$ describing the non-spike related part of $V$. Similarly to (27) the average (15) with $p=q=0$ then reads

$$
\tilde{\rho}(f)=\left\langle V-E_{\mathrm{A}}\right\rangle_{T, w}=\frac{1}{T}\left(\int_{0}^{T_{S}} \tilde{w}(t) V_{S}(t) \mathrm{d} t+\int_{0}^{T} \tilde{w}(t) V_{I S I}(t) \mathrm{d} t\right)-E_{\mathrm{A}} .
$$

As a simplifying hypothesis, let us further assume that $V_{I S I}(t)$ as well as the weight function $w(t)$ obey a scale invariance such that $V_{I S I}(t)=\hat{V}_{I S I}(t f)$ and $w(t)=\hat{w}(t f)$. Substituting $x$ for $t f$ we get

$$
\tilde{\rho}(f) \approx \frac{1}{T} \int_{0}^{T_{S}} w(t) V_{S}(t) \mathrm{d} t+\int_{0}^{1} \hat{w}(x) \hat{V}_{I S I}(x) \mathrm{d} x-E_{\mathrm{A}} .
$$

The first integral covers spike-related phenomena and can be neglected because $w(t)$ is small during the spike and usually $T_{S} \ll T$. According to our assumption, the second integral is independent of $f$, so that $\tilde{\rho}(f)$ is constant. Thus, for this scenario the $\gamma(f)$ term vanishes. A non-zero $\gamma(f)$-term most likely results from a dependence of $V_{I S I}(t)$ on $f$ which does not scale with $f$. Fig. 4 gives one example.

Note that the $\gamma(f)$-term should be independent of $A$ (inset in Fig. 6B). If this is not the case then the weak coupling assumption of the model is invalid.

\subsection{Time constants of adaptation}

In addition to the onset $f$ - $I$-curve, the steady-state $f$ - $I$-curve, and the $\gamma(f)$-term, we still need to know how to measure the adaptation time constant $\tau$ in equation (8b) in order to apply the adaptation model to experimental data. To address this issue we explore the relation between $\tau$ and the effective time constant $\tau_{\text {eff }}$ describing the decay of the 

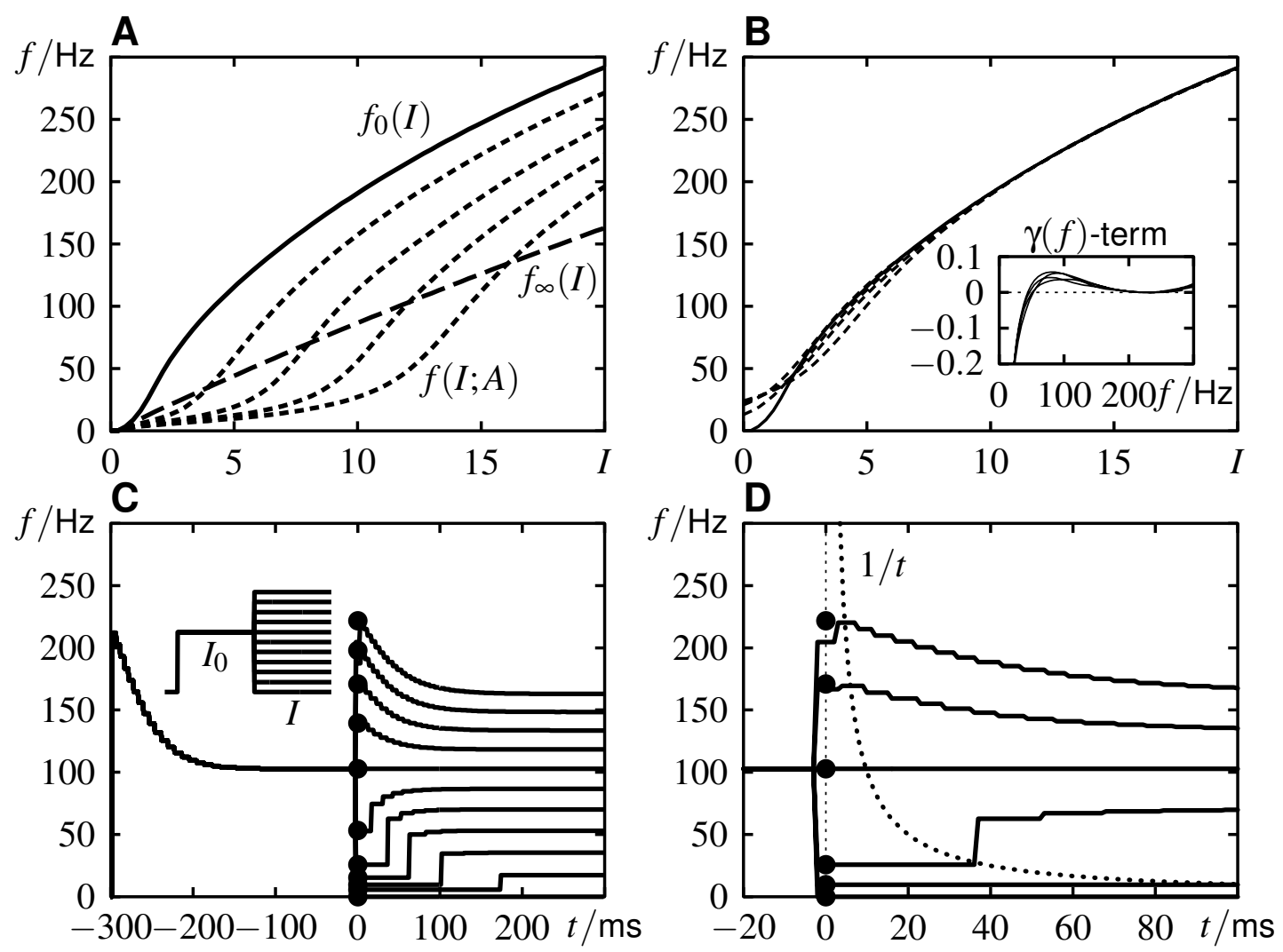

Figure 6: Adapted $f$-I-curves. A Comparison of some adapted $f$-I-curves $f(I ; A)$ with the onset $f$-I-curve $f_{0}(I) \equiv f(I ; 0)$ and the steady-state $f$-I-curve $f_{\infty}(I)$ for the modified Traub-Miles with mAHP-current. B The adapted $f$-I-curves shifted on top of the onset $f$ - $I$-curve so that they align at $f=230 \mathrm{~Hz}$. As expected, all these $f$-I-curves are similar in shape. The inset shows the corresponding $\gamma(f)$-terms which were computed using (28) and $A$ set to the distance of the $f$ - $I$-curves at $f=230 \mathrm{~Hz}$. The $\gamma(f)$-terms are very similar, thus verifying the weak coupling assumption of the universal model. Above a firing frequency of approximately $50 \mathrm{~Hz}$ the $\gamma(f)$ term is small (less than 6\%). The deviations of the $f$-I-curves and thus the high (negative) values of the $\gamma(f)$-term below $50 \mathrm{~Hz}$ may arise due to the difficulties to measure the adapted $f-I$ curves. C Firing frequencies evoked by the protocol for measuring an adapted $f$ - $I$-curve (inset). In this example the neuron is first adapted to $I_{0}=12$ (conditioning stimulus, $-300 \mathrm{~ms}<t<0$ ). Then the input is stepped to different test stimuli $I(t>0)$ to measure the initial response of the adapted neuron at these intensities (dots). The responses to higher test intensities show sharp peaks which decay back to a new steady-state. Lower test intensities result in decreased responses, which increase due to recovery from adaptation to the corresponding steady-state values. D A closer look at some of the responses in $\mathrm{C}$ reveals that the initial responses for stimulus intensities below the conditioning stimulus are not well defined. Since the neuron responds with repetitive firing to the conditioning stimulus, there might be a spike at $t=0$ or shortly before. Thus, the lowest firing frequency that can be measured before a spike at time $t$ is $f \approx 1 / t$ (dotted line). As a consequence, firing frequencies (dots) measured below the $1 / t$-line overestimate the real response. This results in the tails of the adapted $f$-I-curves in A. 
firing frequency during constant stimulation (see Fig. 1B). First we discuss why these two time constants differ in general. Then we investigate how the time course of the adaptation state can be measured and used to estimate $\tau$. Finally, we linearize the model (8) to give a direct relation between $\tau$ and $\tau_{\text {eff }}$.

The different estimates of $\tau$ are illustrated in Fig. 7. For simplicity the $\varepsilon(f)$-term introducing a dependence of the adaptation time constant on the firing frequency is neglected in the following analysis. We justify this in the last paragraph of this section.

In general the adaptation time constant $\tau$ is not identical with the effective time constant $\tau_{\text {eff }}$ (see Fig. 1D). The main reason for this is that the steady-state strength of adaptation $A_{\infty}$ depends on the actual firing frequency. Thus $A_{\infty}$ is not constant and $A(t)$ is not necessarily an exponential function with time constant $\tau$. The time constant $\tau_{A}$, which we obtain by fitting a single exponential to the time course of $A(t)$, may therefore differ from $\tau$. A possible discrepancy between $\tau$ and $\tau_{\text {eff }}$ may also be due to the onset $f$-I-curve and the $\gamma(f)$-term. Both determine how $A$ influences $f$. If $\gamma(f)$ is non-zero or the onset $f$-I-curve is non-linear, $\tau_{\text {eff }}$ differs from $\tau_{A}$ and thus from $\tau$.

Knowing $f_{0}(I)$ and $\gamma(f)$ enables one to calculate the time course of $A$ from (8a):

$$
A=\frac{I-f_{0}^{-1}(f)}{1+\gamma(f)}
$$

Using this equation the time evolution of $A$ can be computed without any knowledge about the adaptation time constant and mechanism, provided $f_{0}^{-1}(I)$ exists. This is guaranteed if $f_{0}(I)$ is strictly monotone in the region of interest but excludes the subthreshold region where $f_{0}(I)$ vanishes. From the decay of $A$ for constant $I$, the corresponding time constant $\tau_{A}$ can be obtained by fitting a single exponential on $A(t)$.

The dependence of $A_{\infty}$ on $f$ still causes $\tau_{A}$ to differ from $\tau$. However, for subthreshold stimuli, $f$ is zero and so is $A_{\infty}$. Equation ( $8 \mathrm{~b}$ ) reduces to $\tau \mathrm{d} A / \mathrm{d} t=-A$, an exponential recovery of $A$ with time constant $\tau$. Since $f=0$, we cannot compute the time course of $A$ directly from (31). Instead, we have to probe $A(t)$ by applying short test stimuli with given intensity $I$ at different times after the offset of an adaptation stimulus. From the onset firing frequencies evoked by these stimuli we can infer $A(t)$ through (31). By fitting a single exponential on $A(t)$ we finally obtain $\tau$. Note, however, that with this method we violate the assumption of high firing frequencies. For $V$ dependent time constants $\tau_{a}(V)$, like the one of the M-type current in the Crook model (Fig. 2B), this method measures the value of the time constant at resting potential which can be much smaller than $\tau$ for the super-threshold regime.

A simple method to estimate $\tau$ for the super-threshold regime is to calculate it di-

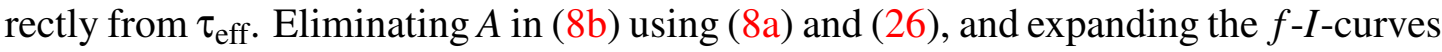



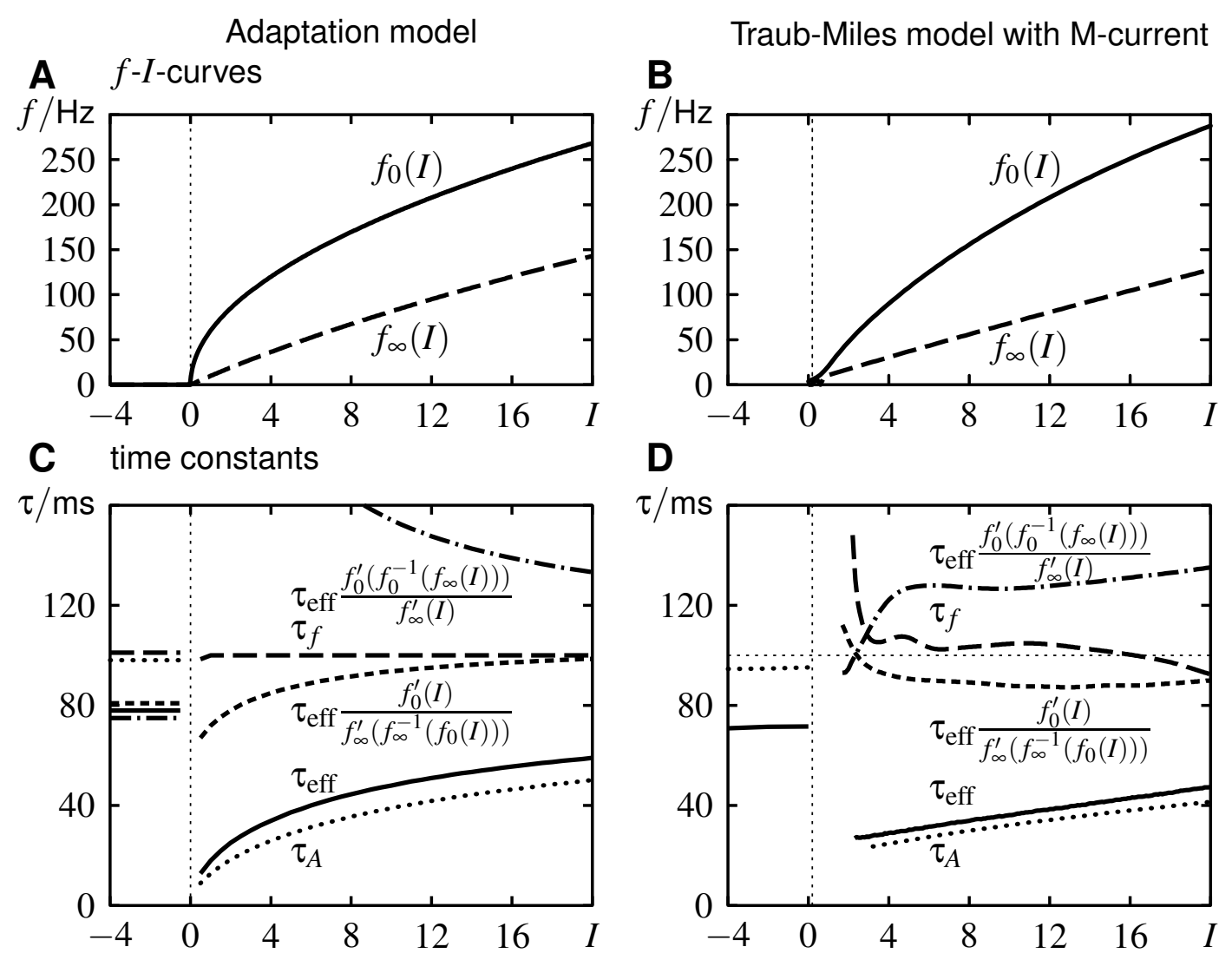

Figure 7: Adaptation time constants. A The onset $f$-I-curve and the steady-state $f$ - $I$-curve used for the simulation of time constants shown in C. The choice $f_{0}(I)=60 \sqrt{I}$ reproduces the shape of a typical $f$-I-curve of a type-I neuron (Ermentrout, 1996) and the corresponding steady-state $f$-I-curve $f_{\infty}(I)=60 \sqrt{I+0.1^{2} 60^{2} / 4}-0.1 \cdot 60^{2} / 2$ results from linear adaptation of medium strength with $A_{\infty}(f)=0.1 \cdot f$. B The $f$-I-curves of the modified Traub-Miles model with M-type current as a more realistic example for estimating the adaptation time constant. C Time constants calculated from $f(t)$ simulated with the model (8) using the $f$-I-curves shown in A and $\tau=100 \mathrm{~ms}$. D Time constants resulting from simulations with the Traub-Miles model where $\tau=100 \mathrm{~ms}$ (horizontal dotted line). C \& D For sub-threshold stimuli $(I<0)$, the time constants were derived from recovery from adaptation as explained in the main text. For superthreshold stimuli $\tau_{\text {eff }}$ is directly measured from $f(t)$ by means of an exponential fit. For suband especially super-threshold stimuli $\tau_{\text {eff }}$ is smaller than $\tau$. The time constant $\tau_{A}$ of the decay of $A(t)$ was computed from the response $f(t)$ using (31). For super-threshold stimuli $\tau_{A}$ differs clearly from $\tau$. The correction of $\tau_{\text {eff }}$ with $f_{0}^{\prime}\left(f_{0}^{-1}\left(f_{\infty}(I)\right)\right) / f_{\infty}^{\prime}(I)$ overestimates $\tau$ whereas the correction with $f_{0}^{\prime}(I) / f_{\infty}^{\prime}\left(f_{\infty}^{-1}\left(f_{0}(I)\right)\right)$ results in values much closer to $\tau$. An alternative way to estimate $\tau$ is to fit $f(t)$ computed with the model (8) to the measured $f(t)$ with $\tau$ as the fit parameter. This gives the best estimate $\tau_{f}$ of the true $\tau$. For sub-threshold stimulus intensities $\tau_{A}$ reveals a good estimate of $\tau$, too. D Note that for low firing frequencies (at about $I<4$ ) the model assumption $f \gg 1 / \tau$ is not fulfilled. 
around $f_{\infty}(I)$

$$
\begin{aligned}
& f_{\infty}^{-1}(f) \approx I+\left.\frac{\mathrm{d} f_{\infty}^{-1}}{\mathrm{~d} f}\right|_{f=f_{\infty}(I)} \cdot\left(f-f_{\infty}(I)\right) \\
& f_{0}^{-1}(f) \approx f_{0}^{-1}\left(f_{\infty}(I)\right)+\left.\frac{\mathrm{d} f_{0}^{-1}}{\mathrm{~d} f}\right|_{f=f_{\infty}(I)} \cdot\left(f-f_{\infty}(I)\right)
\end{aligned}
$$

results in a linear differential equation for $f$

$$
\tau_{\mathrm{eff}}(I) \frac{\mathrm{d} f}{\mathrm{~d} t}=f_{\infty}(I)+\tau_{\mathrm{eff}}(I) f_{0}^{\prime}\left(f_{0}^{-1}\left(f_{\infty}(I)\right)\right) \frac{\mathrm{d} I}{\mathrm{~d} t}-f .
$$

In this equation, $\tau_{\text {eff }}$ is the decay constant of the firing frequency $f$ which is given by

$$
\tau_{\mathrm{eff}}(I)=\tau \frac{f_{\infty}^{\prime}(I)}{f_{0}^{\prime}\left(f_{0}^{-1}\left(f_{\infty}(I)\right)\right)} .
$$

Thus $\tau$ is scaled by the slopes of the $f$-I-curves at the steady-state frequency $f_{\infty}(I)$.

This approximation is correct for small deviations of $f$ from $f_{\infty}(I)$. However, $\tau_{\text {eff }}$ is usually measured by applying a constant stimulus to the unadapted neuron, as in Fig. 1. In this case the initial response deviates significantly from the steady state and dominates the estimate of $\tau_{\text {eff. }}$. It might therefore be better to expand the $f$-I-curves at $f_{0}(I)$ instead of $f_{\infty}(I)$. Doing so we get

$$
\tau_{\mathrm{eff}}(I)=\tau \frac{f_{\infty}^{\prime}\left(f_{\infty}^{-1}\left(f_{0}(I)\right)\right)}{f_{0}^{\prime}(I)}
$$

which generalizes the results of Ermentrout (1998) to arbitrary $f$-I-curves. Especially slightly above the firing threshold of the onset $f$-I-curve $f_{0}(I)$, its slope is much larger than that of the steady-state $f$-I-curve. This causes $\tau_{\text {eff }}$ to be smaller than at higher input intensities. However, the time constant resulting from the M-type current of the Crook model (Fig. 5A) increases for small $f$, thus counteracting the effect of the $f$ - $I$-curves. Inverting (35) allows us to estimate the adaptation time constant $\tau$ from the measured $\tau_{\text {eff }}$ as illustrated in Fig. 7.

An alternative and more precise method to estimate $\tau$ is to fit $f(t)$ computed with the model (8) to the measured $f(t)$ with $\tau$ as the fit parameter. If the resulting $\tau$ depends strongly on the input, one might consider the possible dependence of the time constant on $f$, i.e. $\varepsilon(f)$ may not be negligible.

How strongly might $\tilde{\tau}(f)$ depend on $f$ ? By definition (22) the answer is determined by how $V(t)$ depends on $f$. This is similar to the $f$ dependence arising from averaging the driving force $V-E_{\mathrm{A}}$ (15) discussed earlier (equation (30), see also Fig. 4) with the difference that now the average is not weighted by $w$. Since spikes are short compared 
to the remaining ISI, the main contribution of a possible dependence of $\tilde{\tau}(f)$ on $f$ results from changes of the time course of $V_{I S I}(t)$ which cannot be explained by a simple scaling in time by $f$. Thus, we expect $\tilde{\tau}(f)$ to depend only weakly on $f$. The Crook model (Fig. 5A) confirms this expectation. For firing frequencies higher than $100 \mathrm{~Hz}$ it reaches a constant value. However, for lower firing frequencies it depends on $f$. On the other hand, the time constant of the modified Traub-Miles model is constant by definition.

\section{Signal-transmission properties}

Using the phenomenological model (8) we may now quantify the influence of adaptation on the signal transmission properties of a neuron based solely on the knowledge of its $f$-I-curves and adaptation time constant. Formulating filter properties of a neuron in terms of $f$-I-curves has the important advantage that they can easily be measured with standard current injection techniques. This allows to quantify functional properties of individual neurons with low experimental effort.

There are two different types of $f$-I-curves which have to be distinguished when discussing the signal-transmission properties of a neuron that exhibits adaptation: the adapted $f$-I-curves $f(I ; A)$ including the onset $f$-I-curve $f_{0}(I)=f(I ; 0)$ as a special case on the one hand, and the steady-state $f$ - $I$-curve $f_{\infty}(I)$ on the other hand. In Fig. 6A these different $f-I$-curves are illustrated for the modified Traub-Miles model. The adapted $f$ - $I$-curves describe the instantaneous response of a neuron in a given and fixed adaptation state $A$. They are important for the transmission of stimulus components which are faster than the adaptation dynamics, since only for such stimuli the adaptation state can be considered to be fixed (for more details see section 5.3). Second, there is the steady-state $f$-I-curve $f_{\infty}(I)$. It describes the response of the neuron when it is fully adapted to the applied fixed stimulus intensity, and is therefore the relevant $f$-I-curve for the transmission of stimulus components slower than the adaptation dynamics.

\subsection{Adapted $f$ - $I$-curves}

What do the $f$-I-curves $f(I ; A)$ look like? Neglecting the $\gamma(f)$-term, equation (8a) simplifies to $f=f_{0}(I-A)$. For fixed $A$ the adapted $f-I$-curves are thus obtained by shifting the onset $f$ - $I$-curve by $A$. Adapted $f$-I-curves of the modified Traub-Miles model (Fig. 6A) indeed align on top of the onset $f$-I-curve (Fig. 6B).

We measure adapted $f$-I-curves by first applying a constant stimulus $I_{0}$ to prepare the neuron in a specific adaptation state $A$. We then use different test intensities $I$ and construct the adapted $f$-I-curve from the evoked onset firing frequencies (Fig. $6 \mathrm{C} \& \mathrm{D}$ ). 

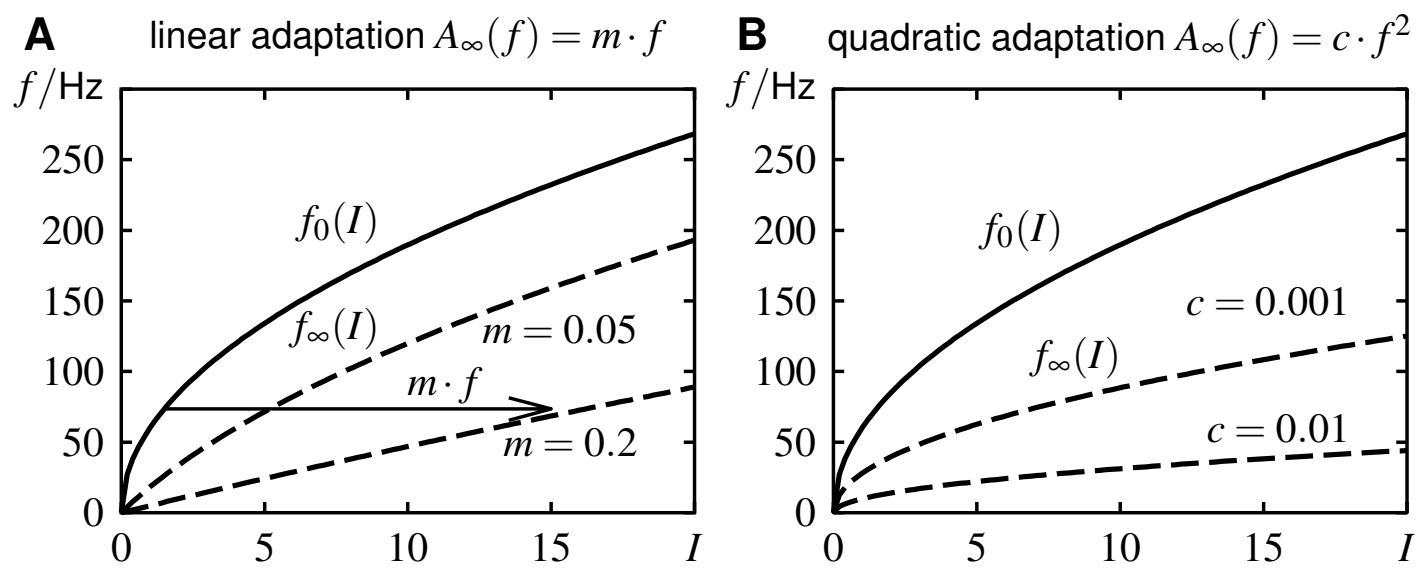

Figure 8: Linearization of the steady-state $f$-I-curve. The effect of adaptation on an onset $f$-I-curve given by $f_{0}(I)=60 \sqrt{I}$ is shown for $\gamma(f)=0$ and $\eta(A)=A$. A Linear adaptation $A_{\infty}(f)=m \cdot f$ linearizes and compresses the steady-state $f$ - $I$-curve. The steady-state $f$ - $I$-curve is approximately linear as long as the firing frequency is so small that the onset $f$ - $I$-curve is close to a vertical line. Adaptation maps each point of the onset $f$-I-curve to the steady-state $f$ $I$-curve by shifting it by the adaptation strength $A_{\infty}(f)$ to higher input values as sketched by the arrow. B With quadratic adaptation $A_{\infty}(f)=c \cdot f^{2}$ the steady-state $f$-I-curves are down-scaled versions of the onset $f$ - $I$-curve. This type of adaptation therefore does not linearize $f_{\infty}(I)$.

\subsection{Linear steady-state $f-I$-curves and linear adaptation}

Alternatively, we can ask which functional form the steady-state $f$ - $I$-curve $f_{\infty}(I)$ has, given a specific dependence of $A_{\infty}$ on $f$. As shown by Ermentrout (1998) adaptation linearizes the $f_{\infty}(I)$-curve. We now generalize his analysis.

In steady state $f$ equals $f_{\infty}(I)$ and $A=A_{\infty}$. From (8a) we obtain the implicit equation

$$
f_{\infty}(I)=f_{0}\left(I-A_{\infty}\left(f_{\infty}(I)\right) \cdot\left[1+\gamma\left(f_{\infty}(I)\right)\right]\right) .
$$

This equation can be generalized if $A$ acts through a function $\eta(A)$. For example, an AHP-type current may depend non-linearly on the calcium concentration, which represents the adaptation state. The implicit equation for $f_{\infty}(I)$ then reads

$$
f_{\infty}(I)=f_{0}\left(I-\eta\left(A_{\infty}\left(f_{\infty}(I)\right)\right) \cdot\left[1+\gamma\left(f_{\infty}(I)\right)\right]\right)=: f_{0}\left(I-\tilde{I}_{A}\left(f_{\infty}(I)\right)\right) .
$$

$\tilde{I}_{A}(f):=\eta\left(A_{\infty}(f)\right) \cdot[1+\gamma(f)]$ generalizes the averaged steady-state adaptation current. Differentiating both sides of (37) yields

$$
\frac{\mathrm{d} f_{\infty}(I)}{\mathrm{d} I}=f_{0}^{\prime}\left(I-\tilde{I}_{A}\left(f_{\infty}(I)\right)\right) \cdot\left(1-\tilde{I}_{A}^{\prime}\left(f_{\infty}(I)\right) \frac{\mathrm{d} f_{\infty}(I)}{\mathrm{d} I}\right),
$$



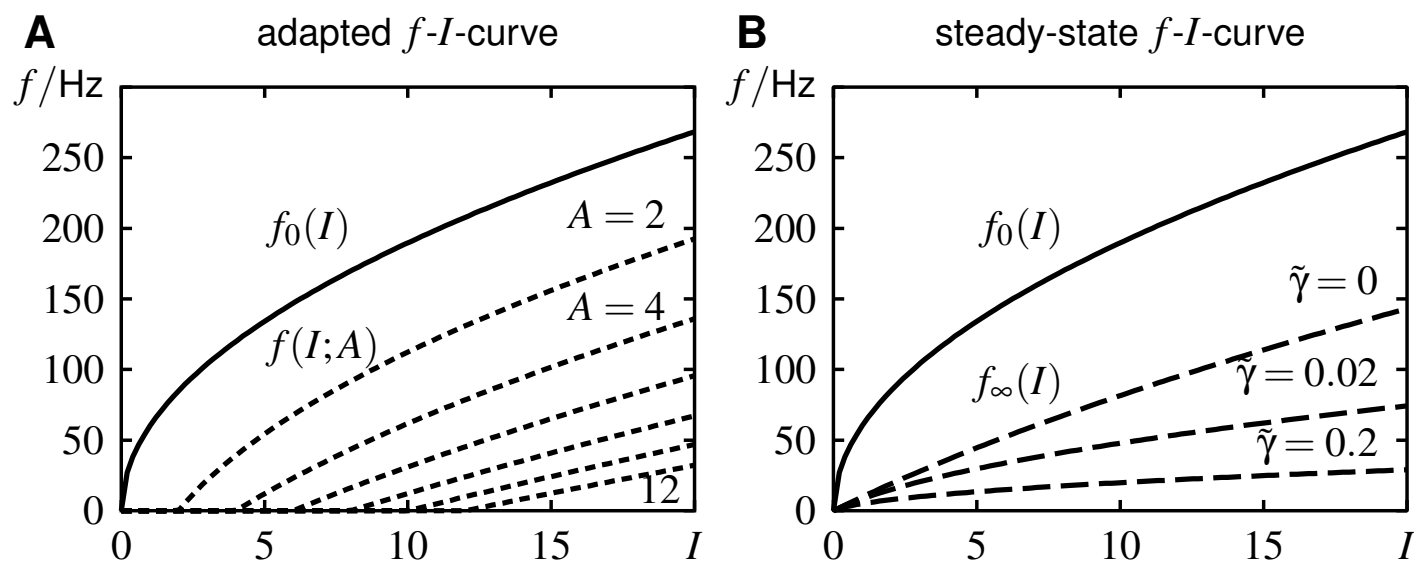

Figure 9: Influence of the $\gamma(f)$-term on $f$-I-curves. A With linear $\gamma(f)=0.02 f$ and increasing adaptation $A=2,4,6,8,10,12$, the adapted $f$ - $I$-curves $f(I ; A)$ are linearized and compressed. B The linearizing effect of linear adaptation $A_{\infty}(f)=0.1 f$ on the steady-state $f$-I-curve $f_{\infty}(I)$ is destroyed by a linear $\gamma(f)$-term $(\gamma(f)=\tilde{\gamma} f, \tilde{\gamma}=0,0.02,0.2)$.

where the prime denotes a derivative with respect to the argument. We obtain

$$
\tilde{I}_{A}^{\prime}\left(f_{\infty}(I)\right)=\frac{1}{f_{\infty}^{\prime}(I)}-\frac{1}{f_{0}^{\prime}\left(I-\tilde{I}_{A}\left(f_{\infty}(I)\right)\right)} .
$$

There are two possibilities to obtain such a linear steady-state $f$-I-curve.

First, the derivative of the onset $f$ - $I$-curve is either constant or infinity. The latter is true for type-I neurons, whose onset $f$-I-curve is a square-root function near their threshold (Ermentrout, 1996). The derivative of $\tilde{I}_{A}$ then also has to be constant. This implies that $\tilde{I}_{A}$ is only allowed to vary linearly with $f$. This is the case if $\gamma(f)$ vanishes and if $\eta\left(A_{\infty}(f)\right)$ depends linearly on $f$. Since most likely $A_{\infty}$ is already proportional to the firing frequency (Fig. 5B), $\eta(A)$ has to equal $A$. We thus obtain

$$
\tilde{I}_{A}\left(f_{\infty}\right)=m \cdot f_{\infty},
$$

where $m$ is a proportionality constant. We refer to this set of conditions as "linear adaptation", since if they are satisfied, $\tilde{I}_{A}$ as well as $A_{\infty}$ depend linearly on $f$. Thus, linear adaptation guarantees a linear steady-state $f$ - $I$-curve for a linear or very steep onset $f$-I-curve. See Fig. 8 and Fig. 9B for illustrations.

The second possibility is that the derivative of the onset $f$-I-curve is neither constant nor infinity. A linear steady-state $f$-I-curve then can still arise if the $\tilde{I}_{A}$ fulfills (39) with $f_{\infty}^{\prime}(I)=$ const and $f_{0}^{\prime}(I)$ calculated from the observed onset $f$-I-curve. We conclude that adaptation may (but need not) linearize the steady-state $f$ - $I$-curve.

In the same manner, we can examine the influence of the $\gamma(f)$-term on the adapted $f$ - $I$-curves. Since $A$ is fixed, the only term introducing an $f$-dependence is $\gamma(f)$ :

$$
f(I ; A)=f_{0}(I-A \cdot[1+\gamma(f)]) .
$$


Taking derivatives of both sides of this equation and rearranging terms results in

$$
\gamma^{\prime}(f) A=\frac{1}{f^{\prime}(I ; A)}-\frac{1}{f_{0}^{\prime}(I-A \cdot[1+\gamma(f)])} .
$$

Analogous to the situation for $f_{\infty}(I)$ (39), there are two cases for getting a linear adapted $f$-I-curve. First, if the onset $f$ - $I$-curve is either a straight line or has an infinite slope at threshold then $\gamma(f)$ must depend linearly on $f$ (see Fig. 9). Note that in this scenario linear steady-state $f$-I-curves are not possible. Second, if the onset $f$-I-curve is neither a straight line nor has an infinite slope, then the $\gamma(f)$-term must depend appropriately on $f$ according to (42) with $f^{\prime}(I ; A)$ only depending on $A$. In this case linear steadystate $f$-I-curves are unlikely since at the same time $\gamma(f)$ and $\tilde{I}_{A}$ have to satisfy equation (42) and equation (39), respectively.

From a different point of view, we may summarize these findings as follows, given a type-I or linear onset $f$ - $I$-curve. Observing a linearized steady-state $f$ - $I$-curve implies that $\gamma(f)$ can be neglected and that the averaged adaptation current $\tilde{I}_{A}(f)$ depends linearly on $f$. A nonlinear steady-state $f$-I-curve implies a nontrivial $\gamma(f)$-term or a nonlinear $\tilde{I}_{A}(f)$. If the adapted $f$-I-curves are shifted versions of the onset $f$-I-curve, the $\gamma(f)$-term can be ruled out, and the nonlinear steady-state $f$-I-curve is caused by a nonlinear $\tilde{I}_{A}(f)$. Note, however, that if the slope of the onset $f$-I-curve is neither constant nor infinity, such general statements cannot be made.

\subsection{High-pass filter properties due to adaptation}

Spike-frequency adaptation is responsible for high-pass filter properties, since adaptation currents resemble an inhibitory feedback. By means of the model (8) we can easily quantify these filter properties for a specific neuron from the knowledge of its onset and steady-state $f$ - $I$-curve, and its adaptation time constant.

In essence the model (8) involves linear dynamics. The only nonlinearities are introduced by the $f$ - $I$-curves and the $\gamma(f)$-term. Consider a stimulus $I(t)$ with sufficiently small fluctuations so that the $f-I$-curves can be linearized around the steady-state firing frequency and the $\gamma(f)$-term can be neglected. We then obtain (33), which is linear in $f$ and we can calculate its transfer function $H_{f}(\omega)$ by means of Fourier-transformation.

$$
\left|H_{f}(\omega)\right|=f_{\infty}^{\prime} \sqrt{\frac{1+\left(\omega \tau_{\mathrm{eff}} f_{0}^{\prime} / f_{\infty}^{\prime}\right)^{2}}{1+\omega^{2} \tau_{\mathrm{eff}}^{2}}}
$$

is the gain for each frequency component $\omega / 2 \pi$ of the stimulus. Gain and phase shift of $H_{f}$ are plotted in Fig. 10A \& B.

Mean and low-frequency components of the stimulus up to $\omega \tau_{\text {eff }} \approx 0.2$ are transmitted via the slope of the steady-state $f$-I-curve $\left(\left|H_{f}(0)\right|=f_{\infty}^{\prime}\right)$. Fast fluctuations with 
A Firing frequency $f$

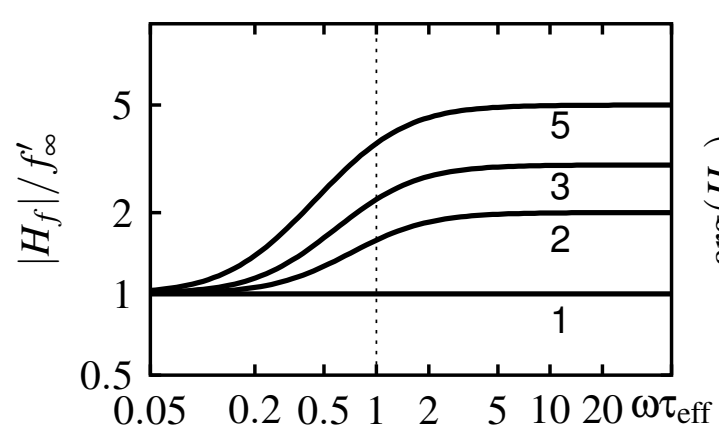

B

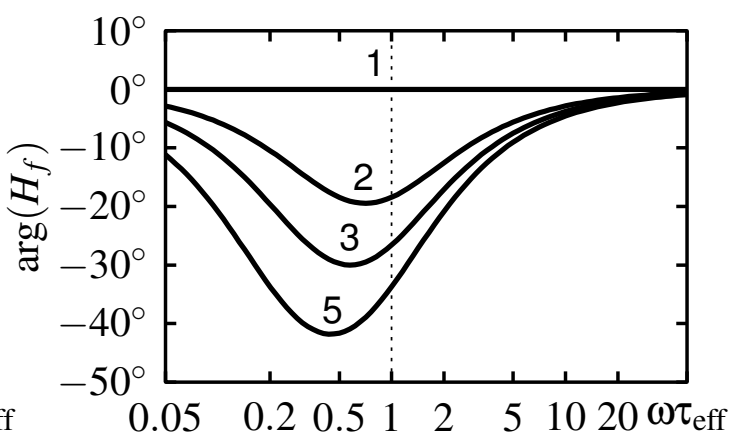

C State of adaptation $A$

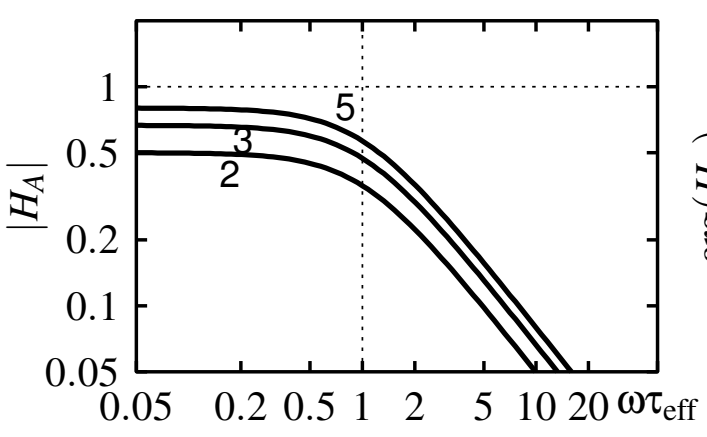

D

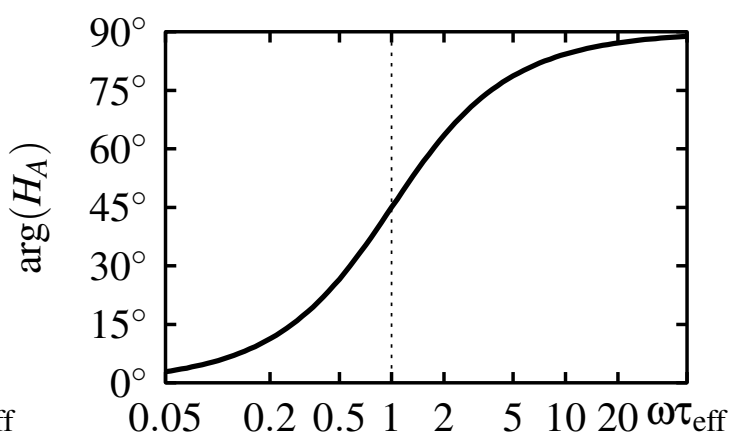

Figure 10: Transfer functions. A Gain and B phase shift of the transfer function for the firing frequency $f(t)$. The gain (43) is plotted as multiples of the slope $f_{\infty}^{\prime}$ of the steady-state $f$-Icurve. At negative phase shifts the output firing frequency advances the input. C Gain and D phase shift of the transfer function for the adaptation state $A(t)$ (44). The gain and the frequency axis are plotted logarithmically. For $\tau_{\text {eff }} \approx 160 \mathrm{~ms}$ the values of the frequency axis correspond to frequency components measured in Hertz. The dotted vertical line marks the cut-off frequency at $\omega \tau_{\text {eff }}=1$. The labels indicate the ratios $f_{0}^{\prime} / f_{\infty}^{\prime}$ of the slopes of the $f$-I-curves.

$\omega \tau_{\text {eff }}>2$ are transmitted much better by the slope of the onset $f$-I-curve $\left(\lim _{\omega \tau_{\text {eff }} \rightarrow \infty}\left|H_{f}(\omega)\right|=f_{0}^{\prime}\right)$. In between at around $\omega_{c} \tau_{\text {eff }}=1$ the firing frequency response shows the strongest phase advance.

This high-pass frequency property of adaptation can be best understood by the dynamics (8b) of the adaptation variable $A$. Substituting $f$ in equation (26) for $A_{\infty}$ by (8a), setting $\gamma(f)=0$ and linearizing at $f=f_{\infty}(I)$ results in

$$
\tau_{\mathrm{eff}} \frac{\mathrm{d} A}{\mathrm{~d} t}=I\left(1-f_{\infty}^{\prime} / f_{0}^{\prime}\right)-A .
$$

The transfer function $H_{A}(\omega)$ of this low-pass filter is shown in Fig. 10C \& D. The adaptation $A$ follows directly the low frequency components $\left(\omega \tau_{\text {eff }}<0.2\right)$ of the stimulus, 
thus shifting the onset $f$ - $I$-curve appropriately towards the corresponding values of the steady-state $f$-I-curve. As a consequence, these slow components are transmitted via the steady-state $f-I$-curve. High frequency components $\left(\omega \tau_{\text {eff }}>2\right)$ have almost no effect on $A$. Thus, fast components are transmitted via an adapted $f$ - $I$-curve, which is the onset $f$-I-curve shifted to higher input intensities because of low frequency components. The shift of the onset $f$-I-curve compensates for the mean value of the stimulus and optimizes the transmission of fast fluctuations, generating a special high-pass filter.

Note that the transfer functions of both $f$ and $A$ depend on the effective time constant $\tau_{\text {eff }}$ and not on the adaptation time constant $\tau$. $\tau_{\text {eff }}$ usually is a function of the input $I$, as shown in the context of Fig. 7. This may result in cut-off frequencies much higher than expected from the value of the adaptation time constant. The dynamical behavior of an adapting neuron is therefore determined by the combined effects of the relative slopes of the onset and steady-state $f-I$-curves and the adaptation time constant $\tau$.

\section{Combining adaptation and spike-generation}

The model (8) simply maps the stimulus $I(t)$ through the onset $f$-I-curve $f_{0}(I)$ into a firing frequency $f(t)$ for a description of the spike generator. This approach is valid as long as the input current $I(t)$ is approximately constant during an interspike interval.

As a consequence of this simple mapping, $f(t)$ fluctuates as fast as $I(t)$ does. However, the transformation of a stimulus into a sequence of spikes acts like a low-pass filter: Given the spikes only, fluctuations of the stimulus $I(t)$ between two succeeding spikes cannot be observed. Thus, the firing frequency $v(t)$ measured from the spikes as the reciprocal of the interspike intervals is in general different and varies more slowly than the model's $f(t)$. Only for stimuli which are approximately constant between two spikes $f(t)$ approaches $v(t)$.

For more rapidly varying stimuli, $f(t)$ has to be fed into a model generating spikes from which a firing frequency $v(t)$ can be calculated and compared with the firing frequency measured experimentally.

The simplest way to do this is to use a non-leaky phase oscillator. This is the canonical model of dynamical systems having a stable limit cycle, just as a spike generator in its super-threshold regime for constant stimuli (Hoppensteadt \& Izhikevich, 1997). Here we apply it to time-dependent stimuli:

$$
\begin{aligned}
\frac{\mathrm{d} \varphi}{\mathrm{d} t}=f(t) & ; \varphi<1 \\
\varphi & =0 \quad ; \varphi=1 \rightarrow \text { spike }
\end{aligned}
$$

The activity $f(t)=f_{0}(I-A[1+\gamma(f)])$ from the adaptation model (8) is the velocity of the phase angle $\varphi$. Every time $\varphi$ reaches unity a cycle is completed and a spike elicited.

We can also use the phase oscillator (45) to compute a continuous firing frequency $v(t)$. At each time $t$ we integrate the activity $f$ symmetrically both back- and forward 
in time until the integral reaches the value one:

$$
\int_{t-\frac{1}{2} T(t)}^{t+\frac{1}{2} T(t)} f\left(t^{\prime}\right) \mathrm{d} t^{\prime}=1 .
$$

The reciprocal of the required integration time $T$ is the desired firing frequency $v(t)$. Dividing this equation by $T$ results in an implicit equation for $v(t)$ as a running average with variable time window $T(t)=1 / v(t)$ :

$$
\mathrm{v}(t)=\frac{1}{T(t)} \int_{t-\frac{1}{2} T(t)}^{t+\frac{1}{2} T(t)} f\left(t^{\prime}\right) \mathrm{d} t^{\prime}
$$

Computing $v(t)$ using (46) captures a large fraction of the low-pass properties of a spiking neuron, but of course this is only a simple sketch of a real spike generator.

To extend our general approach to lower firing frequencies and stronger adaptation currents, it is necessary to incorporate the interaction between adaptation and spike generation. Let

$$
\frac{\mathrm{d} \vec{x}}{\mathrm{~d} t}=\vec{g}(\vec{x}, I(t))
$$

be the dynamics of a specific spike generator, i.e. a $N$-dimensional system of differential equations, which is driven by the input current $I(t)$. Whenever one of the variables $\vec{x}(t)$ (e.g. the membrane potential, or a phase angle) crosses a threshold, there is a spike and this variable may be reset. This is a general formulation of conductance-based models (9), integrate-and-fire models and phase oscillators (45). For the $\theta$-model (Ermentrout, 1996), for example, $\vec{x}=\theta$ and $\vec{g}(\vec{x}, I(t))=q(1-\cos \theta)+(1+\cos \theta) c\left(I(t)-I^{*}\right)$, where $q$ and $c$ are constants and $I^{*}$ is the input intensity at which the bifurcation from quiescence to repetitive firing occurs. Whenever the phase angle $\theta$ crosses $\pi$ there is a spike and $\theta$ is reset to $-\pi$. In order to use the adaptation model (8) in conjunction with (48) we need to go back to the general adaptation current (1) by undoing the averages but keeping the parameterization.

An intermediate approach for moderate adaptation currents is

$$
\begin{aligned}
\frac{\mathrm{d} \vec{x}}{\mathrm{~d} t} & =\vec{g}(\vec{x}, I(t)-A(t) \cdot[1+\gamma(\mathrm{v})]) \\
\tau \cdot[1+\varepsilon(\mathrm{v})] \frac{\mathrm{d} A}{\mathrm{~d} t} & =\frac{A_{\infty}(\mathrm{v})}{\mathrm{v}} \delta\left(t-t_{i}\right)-A
\end{aligned}
$$

where $\delta\left(t-t_{i}\right)$ is Dirac's delta-function, $t_{i}$ is the time of the last spike, and $v=1 /\left(t_{i}-\right.$ $\left.t_{i-1}\right)$ is the instantaneous firing frequency. For the $\theta$-model example this equation reads 


$$
\begin{aligned}
\frac{\mathrm{d} \theta}{\mathrm{d} t} & =q(1-\cos \theta)+(1+\cos \theta) c\left(I-A-I^{*}\right) \\
\tau \frac{\mathrm{d} A}{\mathrm{~d} t} & =s \delta\left(t-t_{i}\right)-A,
\end{aligned}
$$

where we set $\gamma(v)=0, \varepsilon(v)=0$, and $A_{\infty}(v) / v=s=$ const to emphasize the linear character of such an adaptation current. All parameters $\gamma, \tau, \varepsilon$, and $A_{\infty}$ of the adaptation current in (49) and (50) are equal to those of the universal model (8) and thus can be easily measured. However, a model like (49) is still limited to adaptation that is weakly coupled to the spike generator.

To overcome this limitation we have to give up the independence of the adaptation model from microscopic properties of specific adaptation mechanisms. Consider

$$
\begin{aligned}
\frac{\mathrm{d} \vec{x}}{\mathrm{~d} t} & =\vec{g}(\vec{x}, I(t)-y \cdot \tilde{\rho}(\vec{x})) \\
\tau \cdot[1+\varepsilon(v)] \frac{\mathrm{d} y}{\mathrm{~d} t} & =\frac{y_{\infty}(v)}{v} \delta\left(t-t_{i}\right)-y .
\end{aligned}
$$

The adaptation variable $y=\bar{g}_{\mathrm{A}} c a$ is proportional to the adaptation gating variable $a$. $\tilde{\rho}(\vec{x})=m^{p} h^{q}\left(V-E_{\mathrm{A}}\right)$ covers the coupling of the adaptation current on the variables $\vec{x}=$ $(V, m, h, \ldots)$ of the spike generator. This term is no longer independent of the adaptation mechanism. If adaptation is caused by slow recovery from inactivation then $p>0$ or $q>0$. For all other adaptation mechanisms $p=q=0$. The adaptation reversal potential $E_{\mathrm{A}}$ is an additional free parameter. Future studies will show which phenomenological quantities measure this parameter.

The parameterization with macroscopically measurable quantities makes (49) and probably (51) superior to using a standard adaptation current like the M-type current, since all parameters can be estimated from measurements of the firing frequency without the knowledge of the specific adaptation mechanism.

\section{Discussion}

Based on a thorough mathematical analysis of several basic spike adaptation mechanisms, a universal phenomenological adaptation model (8) has been introduced in this paper. Our approach combines three important aspects: biophysics of ionic currents, electrophysiology, and the theory of signal processing. First, the model is derived from well known biophysical kinetics. Second, the model is completely defined by macroscopic quantities such as the neuron's $f-I$-curves and the adaptation time constant. These can be measured easily with standard recording techniques. In particular, neither pharmacological nor voltage-clamp methods are needed, as demonstrated by a recent 
study on the dynamics of insect auditory receptor cells (Benda et al., 2001). Third, the simplicity of the model framework allows quantitative predictions about the signal transmission properties of specific neurons arising from spike-frequency adaptation.

\subsection{Comparison with other adaptation models}

Most modeling studies concerned with spike-frequency adaptation rely on a specific adaptation mechanism. Among these mechanisms the mAHP-current has been investigated intensively. Wang (1998) analyzed a conductance-based model with calcium dynamics and an mAHP-current. He recognized the important difference between the time constant of the calcium removal and the effective time constant as measured from the exponential decay of the firing frequency. However, since a linear model is used, the relation between these two time constants depends on the $f$-I-curves at a given intensity ("percentage adaptation of firing frequency"). This neglects the fact that the investigated type of adaptation depends on the firing frequency and not on input intensity. In a more general investigation, Ermentrout (1998) observed the linearization of steady-state $f$-I-curves in type-I neurons. He compared this result with simulations of a conductance based model with both M-type and mAHP-currents. For $f$-I-curves of the form $f_{0}(I)=c \sqrt{I}$ he derived a relation between $\tau$ and $\tau_{\text {eff }}$ in agreement with the more general equation (35). Adaptation in integrate \& fire models often has been introduced by an adaptive threshold (MacGregor \& Oliver, 1974; Liu \& Wang, 2001). However, such thresholds may result in divisive adaptation instead of the subtractive characteristic of (8a). Quantitative differences between an adaptive threshold and an adaptation current were studied by Liu \& Wang (2001) in leaky integrate \& fire neurons.

The adaptation model introduced by Izhikevich (2000) is a specific implementation of equation (49) for the $\theta$-neuron, which is upto a different scaling of variables identical to the example (50). It assumes a constant adaptation time constant, a steady-state adaptation strength that is proportional to the firing frequency, and a constant driving force, which is independent of the model's phase variable. This model represents the essential properties of moderate adaptation within the canonical model for type-I neurons (Ermentrout, 1996). Thus, it is well suited to investigate adaptation effects for interspike-intervals that are similar to or even longer than the adaptation time constant.

In the model (8) presented here the $\gamma(f)$ and the $\varepsilon(f)$-terms introduce a novel frequency dependence of the averaged adaptation current and time constant, respectively.

\subsection{Model assumptions}

The basic assumption behind the model (8) is that the firing frequency is high compared to the inverse adaptation time constant. This is important for separating adaptation from spike generation (Cartling, 1996; Wang, 1998). Since typical adaptation time constants are larger than $50 \mathrm{~ms}$, the corresponding critical firing frequency is at most $20 \mathrm{~Hz}$. For 
peripheral neurons and regular spiking cells in the cortex (Connors \& Gutnick, 1990) this is not a critical restriction. However, many central neurons fire only rarely so that the interplay of the adaptation current with the spike generator becomes crucial. Both processes have to be analyzed in combination, for example based on the framework of (49) and (51), and specific properties of the spike generator have to be taken into account.

The second main assumption is that fluctuations of the adaptation current do not strongly influence the time course of the spike dynamics. This allows one to replace the adaptation current and the adaptation time-constant and steady-state variable by averages (16), (22), and (23), respectively, only depending on firing frequency. The validity of this weak coupling assumption depends on the properties of the specific spike generator, and is confirmed if the $\gamma(f)$-term does not vary strongly with the adaptation state.

This assumption does not interfere with the switch from type-I to type-II dynamics induced by activation of M-type currents at low potentials, as pointed out by Ermentrout et al. (2001). Our description of the unadapted neuron in terms of its onset $f$-I-curve already includes this effect. In fact, since the gating variable of the M-type current obeys a linear differential equation and enters the current linearly, we can replace it by a sum of two variables. One variable covers the M-type current activated by the low potentials at rest and between spikes, while the other variable is activated during spikes only. The first current is part of the spike generator, contributes to the onset $f$-I-curve and the offset of $\tilde{\rho}(f)$ of the Crook-model, as shown in Fig. 5B, and may alter a type-I neuron into a type-II. Only the second variable induces spike-frequency adaptation.

Following Kirchoff's law, ionic currents are additive in the membrane equation (9). Therefore adaptation caused by ionic currents is subtractive, i.e. the adapted $f$-I-curves are shifted versions of the onset $f$-I-curve. Adaptation may involve separate currents like M-type or AHP-type currents which obviously are additive in the membrane equation. Mechanisms acting via additional gating variables, like the slow inactivation of the sodium current, result also in an additive current, provided the other gating variables involved operate on a faster time scale. The situation is different if an adaptation process modulates the dynamics of an ionic current. For example, the level of intracellular calcium influences gene expression, and could thus slowly modulate ionic currents and eventually change the shape of the $f$-I-curve (Shin et al., 1999; Stemmler \& Koch, 1999).

We have shown that averaging the driving force of the adaptation current results in a constant term $\rho$ plus higher order terms $\gamma(f)$ in the firing frequency $f$. This finding is independent of using Ohm's law, the Goldman-Hodgkin-Katz equation, or other models for membrane currents (Johnston \& Wu, 1997), since we have only exploited the fact that the driving force depends on the membrane potential.

We have also assumed that the adaptation current is linearly scaled by the adaptation variable. Unlike the Hodgkin-Huxley channels, all models of the kinetics of voltage- 
gated adaptation currents are indeed linear (Edman et al., 1987; Fleidervish et al., 1996; Crook et al., 1998; Delord et al., 2000). However, the steady-state mAHP-current may depend non-linearly on the intracellular calcium concentration, as discussed below.

In principle, adaptation could be influenced by all biophysical processes present in the investigated cell. In many cases, however, one process is dominant. A single differential equation may then be used to capture the adaptation phenomena. Faster processes can be included into the spike generator, slower processes can be neglected, and processes with similar time scales can often be combined with this single differential equation. However, it is quite common that the time scales of the adaptation mechanisms depend on the membrane potential or calcium concentration. A single differential equation might then no longer be sufficient to describe adaptation. To our knowledge, no single current with two similar time constants exist (Hille, 1992). However, regarding adaptation due to AHP-type currents several differential equations might indeed be involved. Another likely possibility is that several adaptation currents with similar time constants are jointly responsible for the macroscopically observed spike-frequency adaptation (Madison \& Nicoll, 1984; Köhler et al., 1996; Xia et al., 1998; Stocker et al., 1999). Their time constants could depend in different ways on the firing frequency, and exclude a description in terms of a single differential equation.

\subsection{Specific biophysical mechanisms}

Channels carrying M-type currents are composed out of KCNQ2, KCNQ3 and KCNQ5 subunits (Wang et al., 1998; Schroeder et al., 2000). It is likely that different combinations of these subunits coexist in a single neuron and that they differ in quantitative aspects of their kinetics, especially in their time constants. This could make more then one differential equation necessary for modeling the resulting spike-frequency adaptation.

The mAHP-type current is a prominent current used for modeling studies (Cartling, 1996; Ermentrout, 1998; Wang, 1998; Liu \& Wang, 2001), and serves as an example for linear adaptation, governed by a single differential equation with fixed time constant. However, in contrast to M-type currents and slow recovery from inactivation various assumptions have to be made to fit mAHP-type currents into this picture.

First, there is a possible nonlinear dependence of the mAHP-current on calcium concentration. As a consequence the adaptation current (mAHP-current) would not be proportional to the adaptation state (calcium concentration). While Ermentrout (1998) and Wang (1998) do not consider this possibility, Engel et al. (1999) argue for an important role of such nonlinearity. As shown by Fig. 8, the shape of the steady-state $f$-I-curves of type-I neurons can be linearized only if adaptation is linear; a nonlinear steady-state $f$ - $I$-curve must result from a nonlinear adaptation and/or the $\gamma(f)$-term. Numerous experimental data from type-I neurons are in agreement with linearized steady-state $f$-I-curves (Koike et al., 1970; Gustafsson \& Wigström, 1981) and suggest 
a linear dependence of the adaptation current on its gating variable. However, a distinct linearizing effect requires the steady-state adaptation current to be strong enough. For experimental $f$ - $I$-curves it is sometimes difficult to distinguish whether the steady-state $f$-I-curve is nonlinear due to weak adaptation or due to a true nonlinear adaptation (see, for example, Madison \& Nicoll, 1984; Lanthorn et al., 1984). Thus, in general a nonlinear dependence of the adaptation current on the adaptation state cannot be ruled out.

Second, the mAHP-gating variable is assumed to be fast enough so that it can be replaced by its steady-state variable. Based on mAHP-channel gating data the study of Hirschberg et al. (1998) hints at long time constants ( $>40 \mathrm{~ms}$ ) of this current at low calcium concentrations. Its dynamics cannot be neglected if this time constant exceeds the time constant of the calcium dynamics, and could dominate the adaptation dynamics at low calcium levels. At higher calcium levels, calcium removal would be the prominent component. This could result in a dependence of the macroscopically observed effective adaptation time constant on the firing frequency, and may even require a second differential equation for the gating variable of the mAHP-current.

Third, the time constant of calcium removal could depend on firing frequency. Calcium imaging shows a decreasing time constant of the mean intracellular calcium level with increasing firing frequency (Schiller et al., 1995; Helmchen et al., 1996). This effect seems to be reproduced by detailed models of the calcium dynamics that include diffusion, pumps and buffering (Engel et al., 1999; Schutter \& Smolen, 1998), and might be important for studies how adaptation influences the spike pattern at firing frequencies similar to the adaptation time constant.

Forth, channels mediating the calcium influx during spikes can inactivate on a time scale of several 10 to $100 \mathrm{~ms}$ (Jaffe et al., 1994; Yamada et al., 1998). This inactivation reduces the calcium influx per spike and thus also the strength of spike-frequency adaptation. Therefore a further differential equation could be necessary to incorporate this process, which would act on the steady-state adaptation strength $A_{\infty}(f)$.

Besides the mAHP-current there exist a slow sAHP-current, which induces afterhyperpolarizations and adaptation on time scales of more than one second (Sah \& Clements, 1999; Stocker et al., 1999). There is an ongoing debate about the biophysical processes responsible (Sah \& Clements, 1999). If the slow kinetics of the channels mediating the sAHP-current is the dominating process, an additional differential equation for the gating of the sAHP-current would be needed.

Slow recovery from inactivation has been observed for many different ionic currents. In sodium currents it causes spike-frequency adaptation, whereas in potassium currents it results in spike-frequency facilitation (Edman et al., 1987; Delord et al., 2000). In contrast to the M-type currents and mAHP-currents, slow inactivation operates on longer time scales of about one second and more (French, 1989; Edman et al., 1987; Fleidervish et al., 1996; Martina \& Jonas, 1997; Delord et al., 2000). From a mechanistic point of view the only difference between spike-frequency adaptation and 
facilitation is that the adaptation state increases the effective input current instead of decreasing it.

Currents such as $I_{Q}$ or $I_{h}$ are activated by hyperpolarization (Halliwell \& Adams, 1982; Maccaferri et al., 1993). With depolarizing inputs these currents deactivate and contribute to spike-frequency adaptation. Their dynamics could be treated in a similar fashion as the dynamics of slow recovery from inactivation.

Sanchez-Vives et al. (2000) report a $\mathrm{Na}^{+}$-activated $\mathrm{K}^{+}$-current. This current induces spike-frequency adaptation on a long time scale (about 3 to 10 seconds). Since no details about the kinetics of this current are known, it remains unclear if it can be described by the adaptation model (8). The situation might be similar to that of the sAHP-current.

Finally, electrogenic pumps have to be considered as another cause of slow adaptation (Sanchez-Vives et al., 2000). Their currents act subtractively on the input and, in their simplest form, obey a single linear differential equation. It is, therefore, likely that such currents are also in agreement with the adaptation model.

In summary, many adaptation currents involved in spike generation fit into our phenomenological approach. Specific cases of adaptation due to mAHP-currents and sAHP-currents might be an exception. Several processes with potentially different time scales are involved in these two types of adaptation, possibly requiring more than one differential equation for a precise description of the resulting spike-frequency adaptation.

\subsection{Functional role of adaptation}

The shape of a neuron's $f$-I-curve is important for its signal-transmission properties. Stimuli below the firing threshold $I_{\text {th }}$ are not transmitted at all, and the slope of the $f$ $I$-curve limits the resolution of input modulations. In adapting neurons the $f$-I-curve is not fixed. As we have shown the onset $f-I$-curve is shifted dynamically by the stimulus. This shift partially compensates for the slow frequency components of the stimulus. Therefore adaptation turns a neuron into a high-pass filter. It is the value of the effective time constant of adaptation (35) and not the time constant of the adaptation dynamics (8b) which separates slow and fast stimulus components. The latter are transmitted via the adapted $f$ - $I$-curves. Since these are often shifted versions of the onset $f$-I-curve, the shape of the onset $f-I$-curve determines the transmission of fast components. Slow components are transmitted via the steady-state $f$ - $I$-curve.

In this context, it should be noted that the observation that adaptation "makes the transfer function of neurons logarithmic" (Engel et al., 1999) refers to the steady-state $f$-I-curve, which is only important for the slow stimulus components. The same holds for the linearizing effect of adaptation on $f$ - $I$-curves discussed by Ermentrout (1998).

The high-pass filter characteristics of adaptation make the response of a neuron approximately independent of the mean stimulus intensity, which is removed by shifting 
the $f-I$-curve. Thus, subtractive adaptation implements intensity invariance. Its fidelity depends strongly on the steady-state $f-I$-curve. For vanishing steady-state activity the intensity invariance is achieved best. Note also that subtractive adaptation does not adapt the neuron's $f$-I-curve to higher order statistics of the stimulus (Brenner et al., 2000).

In order to study neural information transfer (Bialek et al., 1991), broad-band filtered white-noise stimuli with cut-off frequencies $>50 \mathrm{~Hz}$ are widely used. Since effective time constants of spike-frequency adaptation are usually larger than $10 \mathrm{~ms}$, the cut-off frequency of the neuron's transfer function is well below $16 \mathrm{~Hz}$. Thus, most of the stimulus power is above the cut-off frequency of adaptation. After the stimulus onset, the neuron adapts and shortly afterwards the adaptation state stays approximately constant. Therefore using such stimuli does not test adaptation. This could explain a result of French et al. (2001), who explored paired spider mechanoreceptor neurons. One of these neurons is phasic, the other phasic-tonic. Surprisingly, the information transfer measured for these two types of neurons was nearly identical. However, both neurons may differ in their steady-state $f-I$-curve while having similar onset $f$-I-curves. For the broad-band stimuli used in this experiment, only properties of the onset $f$-I-curve contribute to the signal transmission. Therefore the differences in the steady-state $f-I$ curves were not tested by the stimuli, which may have resulted in the reported information rates.

Knowing the neuron's firing threshold is essential for improving stimulus reconstruction (Machens et al., 2001). For stimuli with strong low-frequency components the resulting varying shift of the $f-I$-curve and its firing threshold due to adaptation would deteriorate the reconstruction. Information about the actual $f$-I-curve as provided by the adaptation model (8) could eliminate these effects.

Let us finally note that recent studies show that shunting synaptic input results in a shift of $f-I$-curves. This corresponds formally to an adaptation current with a fixed adaptation state. However, the noisy nature of (balanced) synaptic input counteracts this shift and adds a strong divisive component on the resulting $f-I$-curve (Chance et al., 2002).

\section{Acknowledgment}

We thank Matthias Bethge, Paola Pedarzani, and Lorenzo Cingolani for important discussions and references. We are grateful to Hartmut Schütze, Matthias Hennig, Astrid Franz, Bernhard Ronacher, Tim Gollisch, Christian Machens, Raphael Ritz, Laurenz Wiskott, and Martin Stemmler who contributed to this work with helpful suggestions and experimental evidence. This work was supported by the DFG through GK 120 and Innovationskolleg Theoretische Biologie. 


\section{Appendix}

\section{List of symbols}

(parentheses denote defining equations)

\begin{tabular}{|c|c|}
\hline $\bar{V}$ & membrane potential \\
\hline$I_{\mathrm{X}}=g_{\mathrm{X}}\left(V-E_{\mathrm{X}}\right)$ & $\begin{array}{l}\text { ionic current of type } \mathrm{X} \text { with conductance } g_{\mathrm{X}} \text { and reversal po- } \\
\text { tential } E_{\mathrm{X}}\end{array}$ \\
\hline$a$ & gating variable of an adaptation current \\
\hline$T$ & interspike interval (ISI) \\
\hline$f=1 / T$ & firing frequency \\
\hline I & input current \\
\hline$f_{0}(I)$ & onset $f$-I-curve of the unadapted neuron \\
\hline$f_{\infty}(I)$ & steady-state $f$ - $I$-curve \\
\hline$A$ & adaptation state (17) \\
\hline$f(I ; A)$ & adapted $f$-I-curve for a given adaptation state $A$ \\
\hline$\tilde{\rho}(f)=\rho \cdot[1+\gamma(f)]$ & averaged driving force (15) of the adaptation current \\
\hline$\tilde{\tau}(f)=\tau \cdot[1+\varepsilon(f)]$ & averaged time constant of the adaptation gating variable (22) \\
\hline$\kappa(f)$ & averaged steady-state adaptation variable $a_{\infty(V)}(23)$ \\
\hline$A_{\infty}(f)=\bar{g}_{\mathrm{A}} c \rho \kappa(f)$ & steady-state adaptation strength (26) \\
\hline$\tau_{\text {eff }}$ & $\begin{array}{l}\text { effective adaptation time constant measured from the decay of } \\
\text { the firing frequency evoked by a step-like stimulus }\end{array}$ \\
\hline$\tau_{A}$ & time constant measured from the time evolution of $A$ \\
\hline
\end{tabular}

\section{The neuron's weight function for small adaptation currents}

The super-threshold dynamics of a spike generator may be captured by a one-dimensional phase model. The phase-angle $\varphi$ describes the movement along a limit cycle, as determined by some function $g(\varphi ; I)>0$ that depends on the phase angle and input $I$ :

$$
\frac{\mathrm{d} \varphi}{\mathrm{d} t}=g(\varphi ; I)
$$

Each time the system has cycled once around the limit cycle a spike is elicited. (52) can be expanded at some constant value $I_{0}$ and transformed to a new phase variable $\psi$ via $\mathrm{d} \varphi / \mathrm{d} \psi=g\left(\varphi ; I_{0}\right)$

$$
\frac{\mathrm{d} \psi}{\mathrm{d} t}=1+z\left(\psi ; I_{0}\right) \Delta I(\psi, t),
$$

where $\Delta I(\psi, t)=I(\varphi(\psi), t)-I_{0}$ is a small perturbation. The term

$$
z\left(\psi ; I_{0}\right)=\frac{\left.\frac{\partial g(\varphi(\psi) ; I)}{\partial I}\right|_{I=I_{0}}}{g\left(\varphi(\psi) ; I_{0}\right)}
$$


is the neuron's response function (Ermentrout, 1996).

Let $T$ be the period of the perturbed phase oscillator $(\Delta I \neq 0)$ and $T_{0}$ the period of the unperturbed phase oscillator $(\Delta I=0)$. Integrating (53) over one complete cycle of $\psi\left(0 \leq \psi \leq T_{0}\right)$ and expanding the integrand to first order yields

$$
T \approx T_{0}-\int_{0}^{T_{0}} z\left(\psi ; I_{0}\right) \Delta I(\psi, t) \mathrm{d} \psi,
$$

provided $\left|z\left(\psi ; I_{0}\right) \Delta I(\psi, t)\right| \ll 1$.

In the context of averaging the adaptation current (13) $I_{0}=I-\left\langle I_{A}\right\rangle_{T, w}$ and $T=T_{0}$. We want to show that $T$ is not changed by replacing the original $\Delta I_{A}=I_{A}-\left\langle I_{A}\right\rangle_{T, w}$ by its weighted average $\left\langle\Delta I_{A}\right\rangle_{T, w}$. This is true if the integral of the right-hand side of (55) does not change if we replace $\Delta I=\Delta I_{A}$ by $\left\langle\Delta I_{A}\right\rangle_{T, w}$ :

$$
\int_{0}^{T} z\left(\psi ; I_{0}\right) \Delta I_{A}(\psi) \mathrm{d} \psi \stackrel{!}{=} \int_{0}^{T} z\left(\psi ; I_{0}\right)\left\langle\Delta I_{A}(\psi)\right\rangle_{T, w} \mathrm{~d} \psi
$$

Since the average $\left\langle\Delta I_{A}\right\rangle_{T, w}$ is constant during one period $T$ we obtain

$$
\left\langle\Delta I_{A}\right\rangle_{T, w}=\frac{\int_{0}^{T} z\left(\psi ; I_{0}\right) \Delta I_{A}(\psi) \mathrm{d} \psi}{\int_{0}^{T} z\left(\psi ; I_{0}\right) \mathrm{d} \psi}=: \int_{0}^{T} \tilde{z}\left(\psi ; I_{0}\right) \Delta I_{A}(\psi) \mathrm{d} \psi .
$$

This shows that replacing $\Delta I_{A}$ by the weighted average $\left\langle\Delta I_{A}\right\rangle_{T, w}$ has no effect on $T$ if the weight $w$ is given by the neuron's normalized response function $z$. The weight $w(t)$ in (13) is then given by $\tilde{z}(\psi(t)) \cdot \mathrm{d} \psi(t) / \mathrm{d} t$.

Note that because of the assumption $\left|z\left(\psi ; I_{0}\right) \Delta I_{A}(\varphi, t)\right| \ll 1$ this finding is true in the limit for small adaptation currents only. Numerical simulations of $z \cdot \Delta I$ for the models used in this paper result in values which are larger than one. For such strong adaptation currents the weight $w$ in (13) does not equal $\tilde{z}$, since $\tilde{z}$ applies to small perturbations only. However, the appropriate weight $w$ might still reflect the main qualitative properties of the neuron's response function.

\section{Specification of the conductance-based models}

\section{Modified Traub-Miles model}

The modified Traub-Miles model was introduced by Ermentrout (1998). It is a single compartment model with one sodium, potassium, and calcium current. We added either an M-type current or an mAHP-current to induce spike-frequency adaptation.

$$
C \frac{\mathrm{d} V}{\mathrm{~d} t}=-I_{\mathrm{Na}}-I_{\mathrm{K}}-I_{\mathrm{Ca}}-I_{\mathrm{L}}-I_{\mathrm{M}}-I_{\mathrm{mAHP}}+I
$$


$C=1 \mu \mathrm{F} / \mathrm{cm}^{2}$.

Sodium current: $I_{\mathrm{Na}}=\bar{g}_{\mathrm{Na}} m^{3} h\left(V-E_{\mathrm{Na}}\right), \bar{g}_{\mathrm{Na}}=100 \mathrm{mS} / \mathrm{cm}^{2}, E_{\mathrm{Na}}=+50 \mathrm{mV}$, $\mathrm{d} m / \mathrm{d} t=\alpha_{m}(V)(1-m)-\beta_{m}(V) m, \alpha_{m}(V)=0.32(V+54) /(1-\exp (-(V+54) / 4))$, $\beta_{m}(V)=0.28(V+27) /(\exp ((V+27) / 5)-1), \mathrm{d} h / \mathrm{d} t=\alpha_{h}(V)(1-h)-\beta_{h}(V) h$, $\alpha_{h}(V)=0.128 \exp (-(V+50) / 18), \beta_{h}(V)=4 /(1+\exp (-(V+27) / 5))$.

Potassium delayed-rectifier current: $I_{\mathrm{K}}=\bar{g}_{\mathrm{K}} n^{4}\left(V-E_{\mathrm{K}}\right), \bar{g}_{\mathrm{K}}=80 \mathrm{mS} / \mathrm{cm}^{2}$, $E_{\mathrm{K}}=-100 \mathrm{mV}, \mathrm{d} n / \mathrm{d} t=\alpha_{n}(V)(1-n)-\beta_{n}(V) n$, $\alpha_{n}(V)=0.032(V+52) /(1-\exp (-(V+52) / 5)), \beta_{n}(V)=0.5 \exp (-(V+57) / 40)$. Calcium current: $I_{\mathrm{Ca}}=\bar{g}_{\mathrm{Ca}} s_{\infty}(V)\left(V-E_{\mathrm{Ca}}\right), \bar{g}_{\mathrm{Ca}}=5 \mathrm{mS} / \mathrm{cm}^{2}, E_{\mathrm{Ca}}=120 \mathrm{mV}$, $s_{\infty}(V)=1 /(1+\exp (-(V+25) / 5))$.

Leakage current: $I_{\mathrm{L}}=\bar{g}_{\mathrm{L}}\left(V-E_{\mathrm{L}}\right), \bar{g}_{\mathrm{L}}=0.1 \mathrm{mS} / \mathrm{cm}^{2}, E_{\mathrm{L}}=-67 \mathrm{mV}$.

M-type current: $I_{\mathrm{M}}=\bar{g}_{\mathrm{M}} w\left(V-E_{\mathrm{M}}\right), \bar{g}_{\mathrm{M}}=8 \mathrm{mS} / \mathrm{cm}^{2}, E_{\mathrm{M}}=-100 \mathrm{mV}$, $\overline{\tau_{w}(V) \mathrm{d} w / \mathrm{d} t=} w_{\infty}(V)-w, \tau_{w}(V)=100 \mathrm{~ms}, w_{\infty}(V)=1 /(1+\exp (-(V+20) / 5))$. mAHP-current and calcium dynamics: $I_{\mathrm{mAHP}}=\bar{g}_{\mathrm{mAHP}} q\left(V-E_{\mathrm{mAHP}}\right)$, $\bar{g}_{\mathrm{mAHP}}=4 \mathrm{mS} / \mathrm{cm}^{2}, E_{\mathrm{mAHP}}=-100 \mathrm{mV}, q=[C a] /(30+[C a])$, $\mathrm{d}[\mathrm{Ca}] / \mathrm{d} t=-0.002 I_{\mathrm{Ca}}-0.0125[\mathrm{Ca}]$.

\section{Crook model}

We used the model of Crook et al. (1998) with only the M-type current as an additional example of an adapting neuron. It is a two-compartment model. One compartment corresponds to the soma and is modeled by the membrane equation for the potential $V_{s}$ of the soma. It contains all the voltage dependent currents for the generation of spikes and possible adaptation currents. The other compartment captures the whole dendritic tree, and is described by a linear membrane equation. The potential of the second compartment is denoted by $V_{d}$. Both compartments are coupled by the coupling current $I_{\mathrm{C}}$. Note that the input current $I$ is injected into the soma. Therefore the adaptation currents are still additive to the input current. The resting potential of the Crook model is at $-77 \mathrm{mV}$.

$$
\begin{aligned}
C \frac{\mathrm{d} V_{s}}{\mathrm{~d} t} & =-I_{\mathrm{Na}}-I_{\mathrm{K}}-I_{\mathrm{Ca}}-I_{\mathrm{LS}}-I_{\mathrm{M}}-I_{\mathrm{C}} / P+I / P \\
C \frac{\mathrm{d} V_{d}}{\mathrm{~d} t} & =-I_{\mathrm{LD}}+I_{\mathrm{C}} /(1-P)
\end{aligned}
$$

$C=0.8 \mu \mathrm{F} / \mathrm{cm}^{2}, P=0.05$.

Sodium current: $I_{\mathrm{Na}}=\bar{g}_{\mathrm{Na}} m^{2} h\left(V_{s}-E_{\mathrm{Na}}\right), \bar{g}_{\mathrm{Na}}=221 \mathrm{mS} / \mathrm{cm}^{2}, E_{\mathrm{Na}}=+55 \mathrm{mV}$, $\mathrm{d} m / \mathrm{d} t=\alpha_{m}(V)(1-m)-\beta_{m}(V) m$, $\alpha_{m}(V)=0.32\left(-47.1-V_{s}\right) /\left(\exp \left(0.25\left(-47.1-V_{s}\right)\right)-1\right)$, $\beta_{m}(V)=0.28\left(V_{s}+20.1\right) /\left(\exp \left(\left(V_{s}+20.1\right) / 5\right)-1\right), \mathrm{d} h / \mathrm{d} t=\alpha_{h}(V)(1-h)-\beta_{h}(V) h$, $\alpha_{h}(V)=0.128 \exp \left(\left(-43-V_{s}\right) / 18\right), \beta_{h}(V)=4 /\left(\exp \left(\left(-20-V_{s}\right) / 5\right)+1\right)$. 
Potassium delayed-rectifier current: $I_{\mathrm{K}}=\bar{g}_{\mathrm{K}} n\left(V_{s}-E_{\mathrm{K}}\right), \bar{g}_{\mathrm{K}}=47 \mathrm{mS} / \mathrm{cm}^{2}$, $E_{\mathrm{K}}=-90 \mathrm{mV}, \mathrm{d} n / \mathrm{d} t=\alpha_{n}(V)(1-n)-\beta_{n}(V) n$, $\alpha_{n}(V)=0.59\left(-25.1-V_{s}\right) /\left(\exp \left(\left(-25.1-V_{s}\right) / 5\right)-1\right)$, $\beta_{n}(V)=0.925 \exp \left(0.925-0.025\left(V_{s}+77\right)\right)$.

Calcium current: $I_{\mathrm{Ca}}=\bar{g}_{\mathrm{Ca}} s^{2} r\left(V_{s}-E_{\mathrm{Ca}}\right), \bar{g}_{\mathrm{Ca}}=8.5 \mathrm{mS} / \mathrm{cm}^{2}, E_{\mathrm{Ca}}=+120 \mathrm{mV}$, $\mathrm{d} s / \mathrm{d} t=\alpha_{s}(V)(1-s)-\beta_{s}(V) s, \alpha_{s}(V)=0.912 /\left(\exp \left(-0.072\left(V_{s}-5\right)\right)+1\right)$, $\beta_{s}(V)=0.0114\left(V_{s}+8.9\right) /\left(\exp \left(\left(V_{s}+8.9\right) / 5\right)-1\right), \tau_{r}(V) \mathrm{d} r / \mathrm{d} t=r_{\infty}(V)-r$, $r_{\infty}(V)=\min \left(\exp \left(-\left(V_{s}+60\right) / 20\right), 1\right), \tau_{r}(V)=200 \mathrm{~ms}$.

Soma leakage-current: $I_{\mathrm{LS}}=\bar{g}_{\mathrm{LS}}\left(V_{s}-E_{\mathrm{LS}}\right), \bar{g}_{\mathrm{LS}}=2 \mathrm{mS} / \mathrm{cm}^{2}, E_{\mathrm{LS}}=-70 \mathrm{mV}$.

M-type current: $I_{\mathrm{M}}=\bar{g}_{\mathrm{M}} w\left(V_{s}-E_{\mathrm{K}}\right), \bar{g}_{\mathrm{M}}=10 \mathrm{mS} / \mathrm{cm}^{2}, E_{\mathrm{K}}=-90 \mathrm{mV}$, $\overline{\tau_{w}(V) \frac{\mathrm{d} w}{\mathrm{~d} t}=w_{\infty}}(V)-w, w_{\infty}(V)=1 /\left(\exp \left(-\left(V_{s}+35\right) / 10\right)+1\right)$, $\tau_{w}(V)=92 \exp \left(-\left(V_{s}+35\right) / 20\right) /\left(1+0.3 \exp \left(-\left(V_{s}+35\right) / 10\right)\right)$.

Dendrite leakage-current: $I_{\mathrm{LD}}=\bar{g}_{\mathrm{LD}}\left(V_{d}-E_{\mathrm{LD}}\right), \bar{g}_{\mathrm{LD}}=0.05 \mathrm{mS} / \mathrm{cm}^{2}, E_{\mathrm{LD}}=-70 \mathrm{mV}$. Coupling current: $I_{\mathrm{C}}=\bar{g}_{\mathrm{C}}\left(V_{s}-V_{d}\right), \bar{g}_{\mathrm{C}}=1.1 \mathrm{mS} / \mathrm{cm}^{2}$.

\section{References}

Benda, J., Bethge, M., Hennig, M., Pawelzik, K., \& Herz, A. V. M. (2001). Spikefrequency adaptation: phenomenological model and experimental tests. Neurocomput., 38-40, 105-110.

Bialek, W., Rieke, F., de Ruyter van Steveninck, R. R., \& Warland, D. (1991). Reading a neural code. Science, 252, 1854-1857.

Brenner, N., Bialek, W., \& de Ruyter van Steveninck, R. (2000). Adaptive rescaling maximizes information transfer. Neuron, 26, 695-702.

Brown, D. A. \& Adams, P. R. (1980). Muscarinic supression of a novel voltagesensitive $\mathrm{K}^{+}$current in a vertebrate neuron. Nature, 183, 673-676.

Brown, D. A. \& Griffith, W. H. (1983). Calcium-activated outward current in voltageclamped hippocampal neurones of the guinea-pig. J. Physiol., 337, 287-301.

Cartling, B. (1996). A low-dimensional, time resolved and adapting model neuron. Int. J. Neural Syst., 7, 237-246.

Chance, F. S., Abbott, L. F., \& Reyes, A. D. (2002). Gain modulation from background synaptic input. Neuron, 35, 773-782.

Connors, B. W. \& Gutnick, M. J. (1990). Intinsic firing patterns of diverse neocortical neurons. Trends Neurosci, 13, 99-104.

Crook, S. M., Ermentrout, G. B., \& Bower, J. M. (1998). Spike frequency adaptation affects the synchronization properties of networks of cortical oscillators. Neural Comput., 10, 837-854.

Delord, B., Baraduc, P., Costalat, R., Burnod, Y., \& Guigon, E. (2000). A model study of cellular short-term memory produced by slowly inactivating potassium conductances. J. Comput. Neurosci., 8, 251-273. 
Edman, Å., Gestrelius, S., \& Grampp, W. (1987). Analysis of gated membrane currents and mechanisms of firing control in the rapidly adapting lobster stretch receptor neurone. J. Physiol., 384, 649-669.

Engel, J., Schultens, H. A., \& Schild, D. (1999). Small conductance potassium channels cause an activity-dependent spike frequency adaptation and make the transfer function of neurons logarithmic. Biophys. J., 76, 1310-1319.

Ermentrout, B. (1996). Type I membranes, phase resetting curves, and synchrony. Neural Comput., 8, 979-1001.

Ermentrout, B. (1998). Linearization of $f$-I curves by adaptation. Neural Comput., 10, 1721-1729.

Ermentrout, B., Pascal, M., \& Gutkin, B. (2001). The effects of spike frequency adaptation and negative feedback on the synchronization of neural oscillators. Neural Comput., 13, 1285-1310.

Fleidervish, I. A., Friedman, A., \& Gutnick, M. J. (1996). Slow inactivation of $\mathrm{Na}^{+}$ current and slow cumulative spike adaptation in mouse and guinea-pig neocortical neurones in slices. J. Physiol., 493.1, 83-97.

French, A. S. (1989). Two components of rapid sensory adaptation in a cockroach mechanoreceptor neuron. J. Neurophys., 62, 768-777.

French, A. S., Höger, U., Sekizawa, S.-I., \& Torkkeli, P. H. (2001). Frequency response functions and information capacities of paired spider mechanoreceptor neurons. Biol. Cybern., 85, 293-300.

Gustafsson, B. \& Wigström, H. (1981). Shape of frequency-current curves in CA1 pyramidal clls in the hippocampus. Brain Res., 223, 417-421.

Halliwell, J. V. \& Adams, P. R. (1982). Voltage-clamp analysis of muscarinic excitation in hippocampal neurons. Brain Res., 250, 71-92.

Hansel, D., Mato, G., \& Meunier, C. (1995). Synchrony in excitatory neural networks. Neural Comput., 7, 307-337.

Helmchen, F., Imoto, K., \& Sakmann, B. (1996). Ca ${ }^{2+}$ buffering and action potentialevoked $\mathrm{Ca}^{2+}$ signaling in dendrites of pyramidal neurons. Biophys. J., 70, 10691081.

Hille, B. (1992). Ionic membranes of excitable membranes. Sinauer Associates, Sunderland, Mass., 2. edition.

Hirschberg, B., Maylie, J., Adelman, J. P., \& Marrion, N. V. (1998). Gating of recombinant small-conductance Ca-activated $\mathrm{K}^{+}$channels by calcium. J. Gen. Physiol., $111,565-581$.

Hodgkin, A. L. (1948). The local electric changes associated with repetitive action in a non-medullated axon. J. Physiol., 107, 165-181.

Hodgkin, A. L. \& Huxley, A. F. (1952). A quantitative description of membrane current and its application to conduction and excitation in nerve. J. Physiol., 117, 500-544. Hoppensteadt, F. C. \& Izhikevich, E. M. (1997). Weakly connected neural networks. Applied Mathematical Sciences 126. Springer, New York. 
Izhikevich, E. M. (2000). Neural excitability, spiking, and bursting. Int. J. Bif. Chaos, 10, 1171-1266.

Jaffe, D. B., Ross, W. N., Lisman, J. E., Lasser-Ross, N., Miyakawa, H., \& Johnston, D. (1994). A model for dendritic $\mathrm{Ca}^{2+}$ accumulation in hippocampal pyramidal neurons based on fluorescence imaging measurements. J. Neurophys., 71, 10651077.

Johnston, D. \& Wu, S. M.-S. (1997). Foundations of Cellular Neurophysiology. MIT Press, Cambridge, Massachusetts.

Köhler, M., Hirschberg, B., Bond, C. T., Kinzie, J. M., Marrion, N. V., Maylie, J., \& Adelman, J. P. (1996). Small-conductance, calcium activated potassium channels from mammalian brain. Science, 273, 1709-1714.

Koike, H., Mano, N., Okada, Y., \& Oshima, T. (1970). Repetitive impulses generated in fast and slow pyrammidal tract cells by intracellularly applied current steps. Exp. Brain Res., 11, 263-281.

Lanthorn, T., Storm, J., \& Andersen, P. (1984). Current-to-frequency transduction in CA1 hippocampal pyramidal cells: Slow prepotentials dominate the primary range firing. Exp. Brain Res., 53, 431-443.

Liu, Y.-H. \& Wang, X.-J. (2001). Spike-frequency adaptation of a generalized leaky integrate-and-fire model neuron. J. Comput. Neurosci., 10, 25-45.

Maccaferri, G., Mangoni, M., Lazzari, A., \& DiFrancesco, D. (1993). Properties of the hyperpolarization-activated current in rat hippocampal CA1 pyramidal cells. $J$. Neurophys., 69, 2129-2136.

MacGregor, R. J. \& Oliver, R. M. (1974). A model for repetitve firing in neurons. Kybernetic, 16, 53-64.

Machens, C. K., Stemmler, M. B., Prinz, P., Krahe, R., \& Herz, A. V. (2001). Representation of acoustic communication signals by insect auditory receptor neurons. $J$. Neurosci., 21, 3215-3227.

Madison, D. V. \& Nicoll, R. A. (1984). Control of the repetitive discharge of rat CA1 pyramidal neurones in vitro. J. Physiol., 354, 319-331.

Martina, M. \& Jonas, P. (1997). Functional differences in $\mathrm{Na}^{+}$channel gating between fast-spiking interneurones and principal neurones of rat hippocampus. J. Physiol., 505.3, 593-603.

Reyes, A. D. \& Fetz, E. E. (1993). Two modes of interspike interval shortening by brief transient depolarizations in cat neocortical neurones. J. Neurophys., 69, 1661-1672.

Sah, P. \& Clements, J. D. (1999). Photolytic manipulation of $\left[\mathrm{Ca}_{2+}\right]_{i}$ reveals slow kinetics of potassium channels underlying the afterhyperpolarization in hippocampal pyramidal neurons. J. Neurosci., 19, 3657-3664.

Sanchez-Vives, M. V., Nowak, L. G., \& McCormick, D. A. (2000). Cellular mechanisms of long-lasting adaptation in visual cortical neurons in vitro. J. Neurosci., 20, 4286-4299.

Sandler, V. M. \& Barbara, J.-G. (1999). Calcium-induced calcium release contributes to 
action potential-evoked calcium transients in hippocampal CA1 pyramidal neurons. J. Neurosci., 19, 4325-4336.

Schiller, J., Helmchen, F., \& Sakmann, B. (1995). Spatial profile of dendritic calcium transients evoked by action potentials in rat neocortical pyramidal neurones. $J$. Physiol., 487.3, 583-600.

Schroeder, B. C., Hechenberger, M., Weinreich, F., Kubisch, C., \& Jentsch, T. J. (2000). KCNQ5, a novel potassium channel broadly expressed in brain, mediates M-type currents. J. Biol. Chem., 275, 24089-24095.

Schutter, E. D. \& Smolen, P. (1998). Calcium dynamics in large neuronal models. In Koch, C. \& Segev, I., editors, Methods in neural modeling., pages 211-250. MIT.

Schwindt, P. E. (1973). Membrane-potential trajectories underlying motoneuron rhythmic firing at high rates. J. Neurophys., 36, 434-449.

Shin, J., Koch, C., \& Douglas, R. (1999). Adaptive neural coding dependent on the time-varying statistics of the somatic input current. Neural Comput., 11, 18931913.

Stemmler, M. \& Koch, C. (1999). How voltage-dependent conductances can adapt to maximize the information encoded by neuronal firing rate. Nat. Neurosci., 2, 521-527.

Stocker, M., Krause, M., \& Pedarzani, P. (1999). An apamin-sensitive $\mathrm{Ca}^{2+}$-activated $\mathrm{K}^{+}$current in hippocampal pyramidal neurons. Proc. Natl. Acad. Sci. USA, 96, 4662-4667.

Traub, R. D., Wong, R. K. S., Miles, R., \& Michelson, H. (1991). A model of a CA3 hippocampal pyramidal neuron incorporating voltage-clamp data on intrinsic conductances. J. Neurophys., 66, 635-650.

Wang, H.-S., Pan, Z., Shi, W., Brown, B. S., Wymore, R. S., Cohen, I. S., Dixon, J. E., \& McKinnon, D. (1998). KCNQ2 and KCNQ3 potassium channel subunits: molecular correlates of the M-channel. Science, 282, 1890-1893.

Wang, X.-J. (1998). Calcium coding and adaptive temporal computation in cortical pyramidal neurons. J. Neurophys., 79, 1549-1566.

Xia, X.-M., Fakler, B., Rivard, A., Wayman, G., Johnson-Pais, T., Keen, J., Ishii, T., Hirschberg, B., Bond, C., Lutsenko, S., Maylie, J., \& Adelman, J. (1998). Mechanisms of calcium gating in small-conductance calcium-activated potassium channels. Nature, 395, 503-507.

Yamada, W. M., Koch, C., \& Adams, P. R. (1998). Multiple channels and calcium dynamics. In Koch, C. \& Segev, I., editors, Methods in neural modeling., pages 137-170. MIT. 